\title{
Uncertainty Quantification of Turbulence Model Closure Coefficients for Transonic Wall-Bounded Flows
}

\author{
John Schaefer* \\ Missouri University of Science and Technology, Rolla, MO, 65409 \\ Thomas West ${ }^{\dagger}$ \\ NASA Langley Research Center, Hampton, VA 23681 \\ Serhat Hosder $\ddagger$ \\ Missouri University of Science and Technology, Rolla, MO, 65409 \\ Christopher Rumsey, ${ }^{\S}$ Jan-Renee Carlson, ${ }^{\Uparrow}$ and William Kleb॥ \\ NASA Langley Research Center, Hampton, VA 23681
}

\begin{abstract}
The goal of this work was to quantify the uncertainty and sensitivity of commonly used turbulence models in Reynolds-Averaged Navier-Stokes codes due to uncertainty in the values of closure coefficients for transonic, wall-bounded flows and to rank the contribution of each coefficient to uncertainty in various output flow quantities of interest. Specifically, uncertainty quantification of turbulence model closure coefficients was performed for transonic flow over an axisymmetric bump at zero degrees angle of attack and the RAE 2822 transonic airfoil at a lift coefficient of 0.744. Three turbulence models were considered: the Spalart-Allmaras Model, Wilcox (2006) $k-\omega$ Model, and the Menter Shear-Stress Transport Model. The FUN3D code developed by NASA Langley Research Center was used as the flow solver. The uncertainty quantification analysis employed stochastic expansions based on non-intrusive polynomial chaos as an efficient means of uncertainty propagation. Several integrated and point-quantities are considered as uncertain outputs for both CFD problems. All closure coefficients were treated as epistemic uncertain variables represented with intervals. Sobol indices were used to rank the relative contributions of each closure coefficient to the total uncertainty in the output quantities of interest. This study identified a number of closure coefficients for each turbulence model for which more information will reduce the amount of uncertainty in the output significantly for transonic, wall-bounded flows.
\end{abstract}

*Graduate Student, Department of Aerospace and Mechanical Engineering, Student Member AIAA.

$\dagger$ †erospace Engineer, Vehicle Analysis Branch, Systems Analysis and Concepts Directorate, Member AIAA.

¥Associate Professor of Aerospace Engineering, Department of Aerospace and Mechanical Engineering, Senior Member AIAA.

$\S$ Aerospace Engineer, Computational AeroSciences Branch, Research Directorate, AIAA Fellow.

ฯAerospace Engineer, Computational AeroSciences Branch, Research Directorate, Senior Member AIAA.

" Acting Head, Computational AeroSciences Branch, Research Directorate, Lifetime Member AIAA. 


\section{Nomenclature}

\begin{tabular}{|c|c|c|c|c|c|}
\hline$C_{D}$ & Drag Coefficient & $\sigma$ & $\kappa$, & $c_{b 1}, \quad c_{b 2}$ & \\
\hline$C_{L}$ & Lift Coefficient & $c_{v 1}$, & $c_{w 2}$ & $c_{w 3}, \quad c_{t 1}$ & SA Closure Coefficients \\
\hline$C_{p}$ & Pressure Coefficient & $c_{t 2}$, & $c_{t 3}$ & $c_{t 4}$ & \\
\hline$C_{f}$ & Skin Friction Coefficient & & & & \\
\hline$M$ & Mach Number & $\sigma_{k}$ & $\sigma_{w}$ & $\beta^{*}, \quad \beta_{0}$ & W2006 Closure Coefficients \\
\hline$R e$ & Reynolds Number & $\sigma_{d o}$ & $C_{\text {lim }}$ & $\kappa \quad\}$ & W a voo closure coemcients \\
\hline$T$ & Temperature & & & & \\
\hline$n$ & Number of Uncertain Variables & $\sigma_{k 1}$, & $\sigma_{w 1}$ & $\left.\beta_{1}, \quad \beta_{2}\right)$ & \\
\hline$n_{p}$ & Oversampling Ratio & $\beta^{*}$ & $\sigma_{k 2}$ & $\left.\sigma_{w 2}, \quad \kappa\right\}$ & SST Closure Coefficients \\
\hline$N_{s}$ & Total Number of Samples & $a_{1}$ & & & \\
\hline$p$ & Order of Response Surface Polynomial & & & & \\
\hline$C_{D_{\text {press }}}$ & Drag Coefficient due to Pressure & $u$ & Velocity & & \\
\hline$C_{D_{\mathrm{sf}}}$ & Drag Coefficient due to Skin Friction & $\rho$ & Density & & \\
\hline & & $\nu$ & Molecula & ar Kinematic & Viscosity \\
\hline$\alpha^{*}$ & Stochastic Response Function & $\nu_{t}$ & Turbulen & nt Kinematic & Viscosity \\
\hline$\alpha_{i}$ & Deterministic Component of $\alpha^{*}$ & $\mu$ & Molecula & ar Dynamic V & iscosity \\
\hline$\Psi_{i}$ & Random Variable Basis Functions of $\alpha^{*}$ & $\mu_{t}$ & Turbulen & at Eddy Viscc & sity \\
\hline$x$ & Deterministic Vector & $d$ & Distance & from Field $\mathrm{P}$ & oint to Nearest Wall \\
\hline$\xi$ & Random Variable Vector & $\Omega$ & Vorticity & Magnitude & \\
\hline$D$ & Total Variance & & & & \\
\hline$S_{i_{1} \ldots i_{s}}$ & Sobol indices & & & & \\
\hline
\end{tabular}

\section{Introduction}

Turbulence is one of the greatest unsolved mysteries of classical physics. Due to the highly complex and chaotic nature of the phenomenon, the current physical understanding of turbulence is incomplete. Efforts have been made to create turbulence models to predict turbulent flows by using computational fluid dynamics (CFD); however, the lack of physical knowledge has forced modelers to use dimensional analysis and other methods to identify several constants, called closure coefficients, which balance the model equations. The numerical values of the closure coefficients in current turbulence models are chosen by using a combination of heuristic and empirical decision making. While modelers typically use experimental data to validate their choice of closure coefficients, a turbulence model is not guaranteed to be universally valid for any arbitrary flow.

The goal of this work was to quantify the uncertainty and sensitivity of commonly used turbulence models in Reynolds-Averaged Navier-Stokes (RANS) codes due to uncertainty in the values of closure coefficients for transonic, wall-bounded flows and to rank the contribution of each coefficient to uncertainty in various output flow quantities of interest. Specifically, three turbulence models are considered: the Spalart-Allmaras One-Equation Model (SA), ${ }^{1}$ the Wilcox (2006) $k-\omega$ Two-Equation Model (W2006), ${ }^{2}$ and the Menter ShearStress Transport Two-Equation Model (SST). ${ }^{3}$ Each model features its own unique set of closure coefficients, which are discussed further in Section III.

Fun3D, an unstructured RANS code of NASA Langley Research Center (LaRC), was chosen as the flow solver for all simulations in this work. More information about FUn3D is included in Section II.A. Two CFD problems are considered in this work. The first is an axisymmetric, transonic bump validation case from Bachalo and Johnson, ${ }^{4}$ which is discussed in detail in Section II.B. The second CFD problem is the RAE 2822 transonic airfoil, which is discussed in detail in Section II.C.

To minimize the large computational expense associated with uncertainty quantification of high-fidelity CFD simulations with traditional sampling methods (e.g., Monte Carlo), stochastic expansions based on non-intrusive polynomial chaos (NIPC) are used as an efficient means of uncertainty propagation in this work. ${ }^{5}$ All closure coefficients were treated as epistemic uncertain variables (i.e., uncertainty due to lack of knowledge and not due to inherent, systemic uncertainty). Sobol indices, ${ }^{6}$ which are global nonlinear 
sensitivity indices based on variance decomposition, were used to rank the relative contributions of each closure coefficient to the total uncertainty in the output quantities of interest. For the transonic bump, the output quantities of interest are drag coefficient, pressure coefficient, skin friction coefficient, and separation bubble size. For the RAE 2822, the output quantities of interest are lift coefficient, pressure and skin friction components of drag coefficient, and pressure coefficient.

In Section II, an overview of the flow solver and CFD test problems is presented. In Section III, the details of each of the turbulence models considered here are discussed. A summary of the closure coefficients and their domains is presented for each model. In Section IV, the Uncertainty Quantification (UQ) analysis and computational expense is discussed in further detail. In Section V, the results of all UQ analyses are presented. Finally in Section VI, conclusions are made and some suggestions for future work are presented.

\section{Flow Solver and CFD Test Problems}

In this section, the flow solver and CFD test problems are discussed. First, the details of the selected solver are provided. Next information is given for the freestream conditions and meshes of each CFD test problem.

\section{II.A. Flow Solver}

All flow solutions in this work were obtained by using a modified form of FUN3D version 12.4. ${ }^{7}$ FUn3D is an unstructured, node-based, three-dimensional, finite-volume RANS code capable of solving steady and unsteady laminar or turbulent flows with subsonic to hypersonic speeds. The modifications to the code were performed to allow changes to the values of turbulence model closure coefficients in the uncertainty analyses. All solutions used a Roe, second-order flux differencing scheme with no flux limiter and second-order spatial accuracy for the viscous terms. The CFL numbers for the mean flow and turbulence model equations were ramped from 10 to 50 and from 5 to 30, respectively, across 500 iterations, and then the CFL numbers were fixed at their maximum values until steady-state convergence was achieved.

\section{II.B. Problem 1: Axisymmetric Transonic Bump}

The first CFD problem considered in this work is an axisymmetric transonic bump validation case from Bachalo and Johnson. ${ }^{4}$ The test article in the experiment consisted of a thin-walled cylinder that extended $61 \mathrm{~cm}$ upstream of the bump's leading edge, and the cylinder had an outside diameter of $15.2 \mathrm{~cm}$ with a bump chord of $20.32 \mathrm{~cm}$. The flow has freestream $M=0.875, T=540^{\circ} \mathrm{R}$, and $R e=2.763 \times 10^{6}$ (based on the bump length of $c=1$ ). All flow solutions were obtained by using the $721 \times 321$ grid from the NASA Langley Research Center turbulence modeling resource website. ${ }^{8}$ The mesh contains 462,882 nodes, $1,155,121$ edges, and 230,400 hexahedral cells. It consists of a $1^{\circ}$ wedge rotated about the $\mathrm{x}$-axis. The sides of the mesh are treated as periodic interfaces; they are periodic with each other and rotated through $1^{\circ}$ in order to simulate axisymmetry. The surface of the bump and cylinder has a solid wall boundary condition, and all other boundary conditions are farfield Riemann. A graphical overview of the axisymmetric transonic bump problem is included in Fig. 1. The circles below the mesh in the figure represent the axisymmetry about the $x$-axis. An example pressure contour plot is included in Fig. 2 for the transonic bump case obtained with the SA baseline model.

\section{II.C. Problem 2: RAE 2822 Transonic Airfoil}

The second CFD problem considered in this work is the RAE 2822 transonic airfoil at a lift coefficient of 0.744. The experimental data for this case is from Cook and McDonald. ${ }^{9}$ The flow has freestream $M=0.729, T=540^{\circ} \mathrm{R}$, and $R e=6.5 \times 10^{6}$ (based on the airfoil chord of $c=1$ ). Numerical integration of the experimental pressure coefficient data yields a lift coefficient of $C_{L}=0.744$; the angle of attack was adjusted for each baseline turbulence model to match this value, and all subsequent simulations for each model used the same angle of attack as the one found for the baseline. All flow solutions were obtained by using a $513 \times 161$ grid containing 206,272 nodes, 514,208 edges, and 102,400 hexahedral cells. The grid is a 2D C-mesh extruded one unit in the $y$-direction with $y$-symmetry boundary conditions on either side and with a solid wall boundary condition on the airfoil surface. All other boundary conditions are farfield 


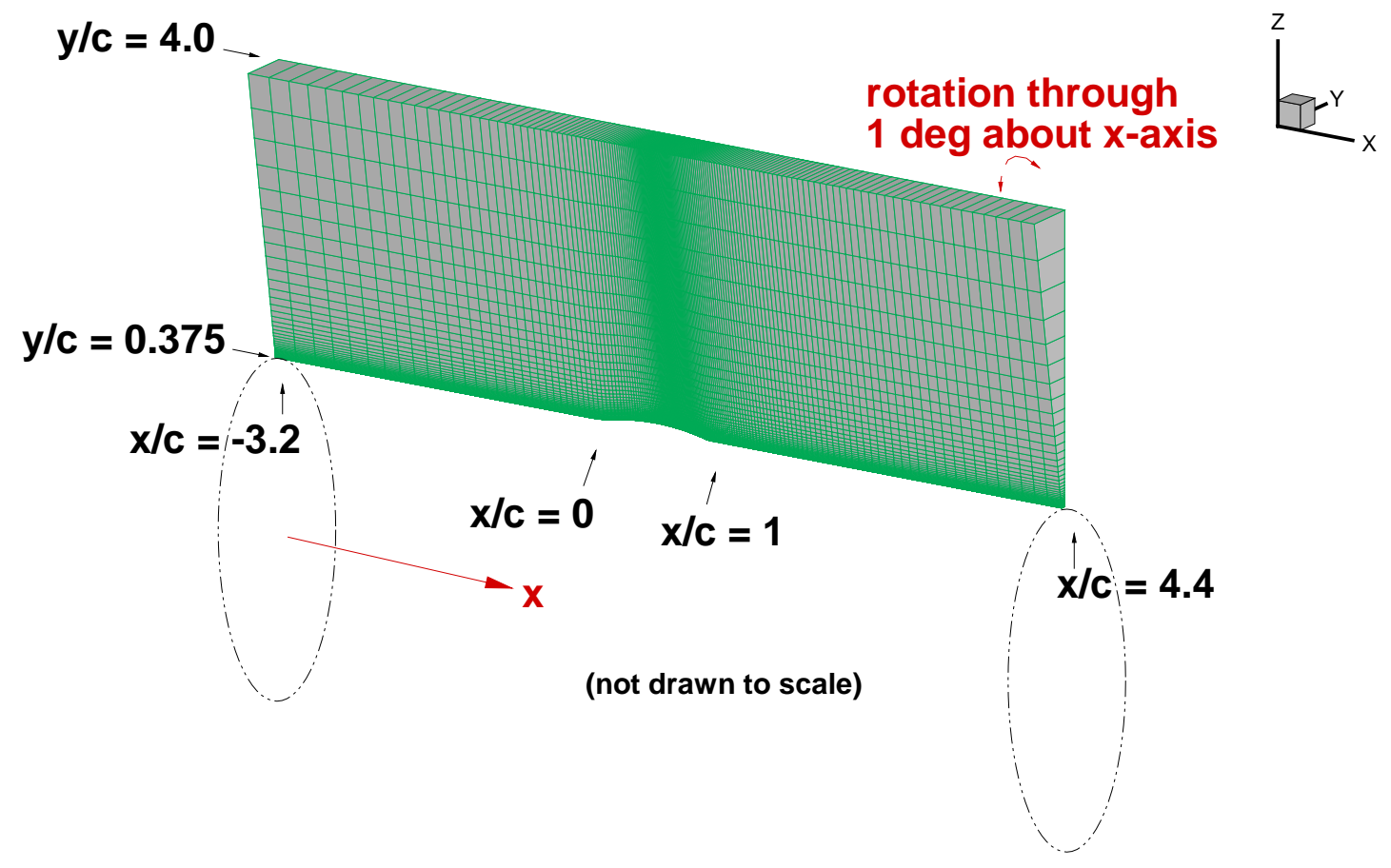

Figure 1. Axisymmetric transonic bump overview from Ref. 8.

Riemann. A graphical view of the RAE 2822 grid is included in Fig. 3. An example pressure contour plot is included in Fig. 4, which was obtained with the SA baseline model.

\section{Turbulence Models}

In this section, the details of the turbulence models employed in this work are outlined. Each subsection contains an overview of a turbulence model, its closure coefficient values, and estimates of the epistemic intervals for each closure coefficient. Note that the two-equation turbulence model equations are written in conserved variable form, but the flow solutions are calculated with primitive variable form. Note that in this work, the nomenclature from the NASA LaRC turbulence modeling website ${ }^{8}$ is used.

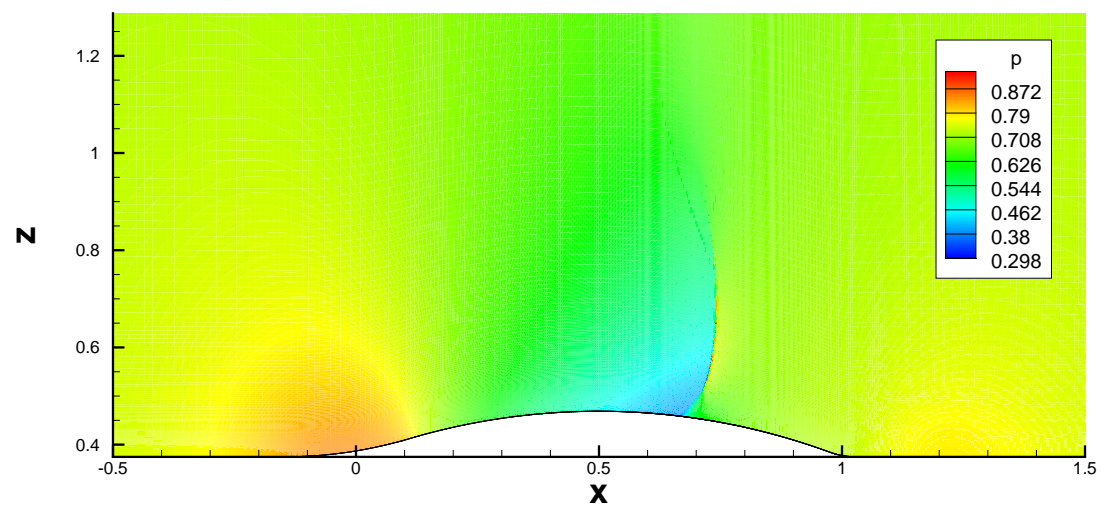

Figure 2. Non-dimensional pressure contours (scaled with the freestream value) of axisymmetric transonic bump. 


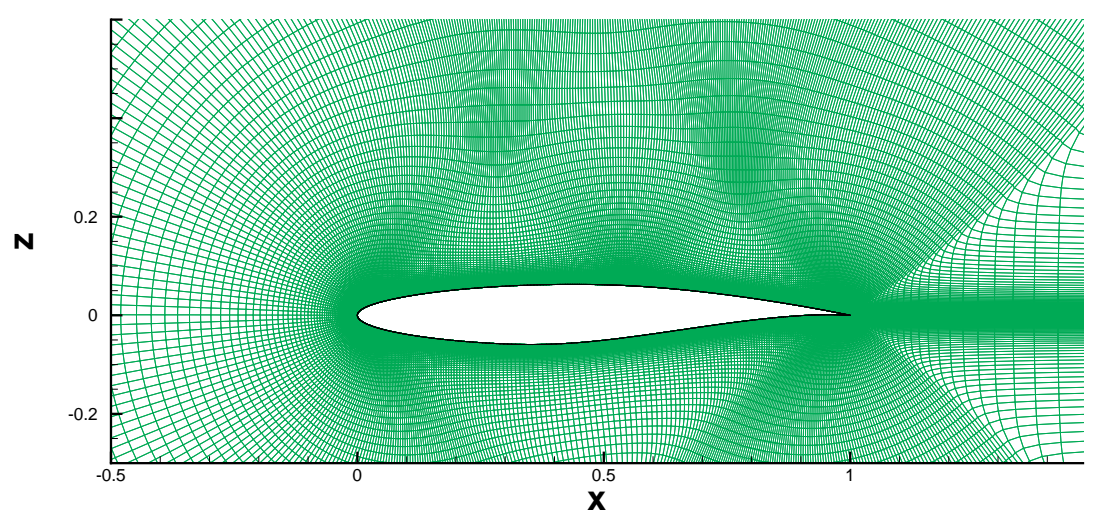

Figure 3. The computational grid around the RAE 2822 transonic airfoil.

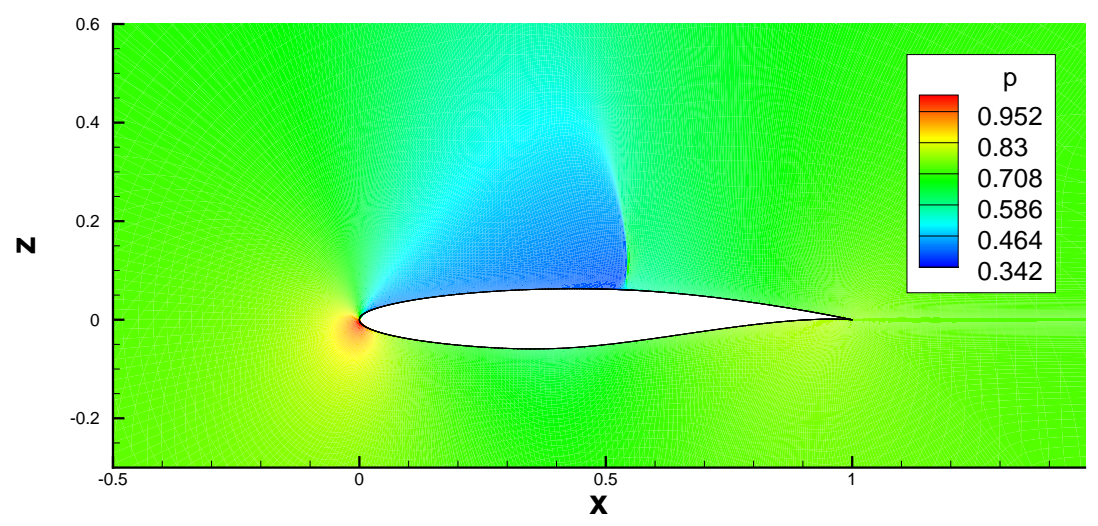

Figure 4. Non-dimensional pressure contours (scaled with the freestream value) of RAE 2822 Airfoil.

\section{III.A. Spalart-Allmaras One-Equation Model (SA)}

The SA model was developed as an evolution of the Nee-Kovasznay model, ${ }^{10}$ but with several near-wall and compressibility corrections. ${ }^{1}$ It is simpler, less computationally expensive, and more computationally robust than multi-equation models, and it is widely used for aerodynamic flows in engineering applications. The model is given by

$$
\frac{\partial \hat{\nu}}{\partial t}+u_{j} \frac{\partial \hat{\nu}}{\partial x_{j}}=c_{b 1}\left(1-f_{t 2}\right) \hat{S} \hat{\nu}-\left[c_{w 1} f_{w}-\frac{c_{b 1}}{\kappa^{2}} f_{t 2}\right]\left(\frac{\hat{\nu}}{d}\right)^{2}+\frac{1}{\sigma}\left[\frac{\partial}{\partial x_{j}}\left((\nu+\hat{\nu}) \frac{\partial \hat{\nu}}{\partial x_{j}}\right)+c_{b 2} \frac{\partial \hat{\nu}}{\partial x_{i}} \frac{\partial \hat{\nu}}{\partial x_{i}}\right]
$$

The full formulation of the model is given by Spalart and Allmaras. ${ }^{1}$ The closure coefficients and their suggested values are given in Table 1 . The closure coefficients also include

$$
c_{w 1}=\frac{c_{b 1}}{\kappa^{2}}+\frac{1+c_{b 2}}{\sigma}
$$

The turbulent eddy viscosity is computed from:

$$
\mu_{t}=\rho \hat{\nu} f_{v 1}
$$

where

$$
f_{v 1}=\frac{\chi^{3}}{\chi^{3}+c_{v 1}^{3}}, \quad \chi=\frac{\hat{\nu}}{\nu}
$$


and $\rho$ is the density, $\nu=\mu / \rho$ is the molecular kinematic viscosity, and $\mu$ is the molecular dynamic viscosity. Additional definitions are given by the following equations:

$$
\hat{S}=\Omega+\frac{\hat{\nu}}{\kappa^{2} d^{2}} f_{\nu 2}
$$

where $\Omega=\sqrt{2 W_{i j} W_{i j}}$ is the magnitude of the vorticity, $d$ is the distance from the field point to the nearest wall, and

$$
\begin{aligned}
f_{v 2} & =1-\frac{\chi}{1+\chi f_{\nu 1}}, \quad f_{w}=g\left[\frac{1+c_{w 3}^{6}}{g^{6}+c_{w 3}^{6}}\right]^{1 / 6}, \quad g=r+c_{w 2}\left(r^{6}-r\right) \\
r & =\min \left[\frac{\hat{\nu}}{\hat{S} \kappa^{2} d^{2}}, 10\right], \quad f_{t 2}=c_{t 3} \exp \left(-c_{t 4} \chi^{2}\right), \quad W_{i j}=\frac{1}{2}\left(\frac{\partial u_{i}}{\partial x_{j}}-\frac{\partial u_{j}}{\partial x_{i}}\right)
\end{aligned}
$$

According to Spalart and Allmaras, ${ }^{1}$ the following constraints should be enforced:

$$
\begin{aligned}
\sigma & \in[0.6,1.0] \\
c_{t 1} & \in[0.1,10] \\
c_{t 3} & >1.0 \\
c_{v 1} & >6.9
\end{aligned}
$$

Spalart and Allmaras also note in Ref. 1 that $c_{t 2}$ is "not a candidate for much adjustment," and that $c_{t 4}$ can be decreased some, but that "values much smaller than 1 would start affecting the results in the turbulent region." Bailey et al. ${ }^{11}$ determined that $\kappa=0.40 \pm 0.02$ in their turbulent pipe flow experiments, and this range of $\kappa$ was used for all of the turbulence models examined in this work. In private communication with the first author, ${ }^{12}$ Spalart recommended using the bounds of Fig. 1 in Ref. 1 to determine the epistemic intervals of $c_{b 1}, c_{b 2}$, and $c_{w 2}$. Spalart also recommended that

$$
c_{w 3} \in[1.75,2.5]
$$

In total, eleven closure coefficients were varied in the UQ analysis for SA. A summary of the SA closure coefficients to be varied, and their associated epistemic intervals are included in Table 1.

\section{III.B. Wilcox (2006) $k$ - $\omega$ Two-Equation Model (W2006)}

The $k-\omega$ model utilizes two equations to model a turbulent kinetic energy and length scale determining variable. The first equation is for turbulent kinetic energy, $k$, and the second equation is for the dissipation per unit turbulence kinetic energy, $\omega$. In 2006, Wilcox introduced a new version of the $k$ - $\omega$ model which

Table 1. SA Closure Coefficients and Associated Epistemic Intervals.

\begin{tabular}{cccc}
\hline Coefficient & Standard Value & Lower Bound & Upper Bound \\
\hline$\sigma$ & $2 / 3$ & 0.6 & 1.0 \\
$\kappa$ & 0.41 & 0.38 & 0.42 \\
$c_{v 1}$ & 7.1 & 6.9 & 7.3 \\
$c_{w 3}$ & 2.0 & 1.75 & 2.5 \\
$c_{t 1}$ & 1.0 & 0.1 & 10.0 \\
$c_{t 2}$ & 2.0 & 1.9 & 2.1 \\
$c_{t 3}$ & 1.2 & 1.0 & 2.0 \\
$c_{t 4}$ & 0.5 & 0.3 & 0.7 \\
$c_{b 1}$ & 0.1355 & 0.12893 & 0.13700 \\
$c_{b 2}$ & 0.622 & 0.60983 & 0.68750 \\
$c_{w 2}$ & 0.3 & 0.05500 & 0.35250 \\
\hline
\end{tabular}


greatly improved its accuracy for free shear flows and strongly separated flows by introducing a cross-diffusion term and a built-in stress-limiter. ${ }^{13}$ The W2006 model is given by

$$
\begin{aligned}
& \frac{\partial(\rho k)}{\partial t}+\frac{\partial\left(\rho u_{j} k\right)}{\partial x_{j}}=P-\beta^{*} \rho \omega k+\frac{\partial}{\partial x_{j}}\left[\left(\mu+\sigma_{k} \frac{\rho k}{\omega}\right) \frac{\partial k}{\partial x_{j}}\right] \\
& \frac{\partial(\rho \omega)}{\partial t}+\frac{\partial\left(\rho u_{j} \omega\right)}{\partial x_{j}}=\frac{\gamma \omega}{k} P-\beta \rho \omega^{2}+\frac{\partial}{\partial x_{j}}\left[\left(\mu+\sigma_{w} \frac{\rho k}{\omega}\right) \frac{\partial \omega}{\partial x_{j}}\right]+\frac{\rho \sigma_{d}}{\omega} \frac{\partial k}{\partial x_{j}} \frac{\partial \omega}{\partial x_{j}}
\end{aligned}
$$

The full formulation of the model is given by Wilcox. ${ }^{2}$ The closure coefficients and their suggested values are given in Table 2 . The closure coefficients also include

$$
\gamma=\frac{\beta_{0}}{\beta^{*}}-\sigma_{w} \frac{\kappa^{2}}{\sqrt{\beta^{*}}}
$$

Definitions of the terms in the model include:

$$
\begin{aligned}
P & =\tau_{i j} \frac{\partial u_{i}}{\partial x_{j}} \\
\tau_{i j} & =\mu_{t}\left(2 S_{i j}-\frac{2}{3} \frac{\partial u_{k}}{\partial x_{k}} \delta_{i j}\right)-\frac{2}{3} \rho k \delta_{i j} \\
S_{i j} & =\frac{1}{2}\left(\frac{\partial u_{i}}{\partial x_{j}}+\frac{\partial u_{j}}{\partial x_{i}}\right)
\end{aligned}
$$

and the turbulent eddy viscosity is computed from:

$$
\mu_{t}=\frac{\rho k}{\hat{\omega}}
$$

where:

$$
\begin{aligned}
\hat{\omega} & =\max \left[\omega, C_{l i m} \sqrt{\frac{2 \bar{S}_{i j} \bar{S}_{i j}}{\beta^{*}}}\right] \\
\bar{S}_{i j} & =S_{i j}-\frac{1}{3} \frac{\partial u_{k}}{\partial x_{k}} \delta_{i j}
\end{aligned}
$$

The auxiliary functions are

$$
\begin{aligned}
& \chi_{\omega}=\left|\frac{\Omega_{i j} \Omega_{j k} \hat{S}_{k i}}{\left(\beta^{*} \omega\right)^{3}}\right|, \quad f_{\beta}=\frac{1+85 \chi_{\omega}}{1+100 \chi_{\omega}} \quad, \sigma_{d}=\left\{\begin{array}{l}
0, \quad \frac{\partial k}{\partial x_{j}} \frac{\partial \omega}{\partial x_{j}} \leq 0 \\
100
\end{array}\right. \\
& \hat{S}_{k i}=S_{k i}-\frac{1}{2} \frac{\partial u_{m}}{\partial x_{m}} \delta_{k i}, \quad \Omega_{i j}=\frac{1}{2}\left(\frac{\partial u_{i}}{\partial x_{j}}-\frac{\partial u_{j}}{\partial x_{i}}\right) \quad \sigma_{d o}, \quad \frac{\partial k}{\partial x_{j}} \frac{\partial \omega}{\partial x_{j}}>0
\end{aligned}
$$

In Ref. 13, Wilcox states that $\tau_{x y} / k \approx 3 / 10$, so $\beta^{*}=\left(\tau_{x y} / k\right)^{2} \approx 9 / 100$. From this description, the author assumed that $\tau_{x y} / k \in[0.28,0.32]$, yielding $\beta^{*} \in[0.0784,0.1024]$. Wilcox also states that $\beta^{*} / \beta_{0}=1.25 \pm 0.06$ and that the following inequalities must hold true:

$$
\begin{aligned}
\sigma_{d o} & >\sigma_{k}-\sigma_{w} \\
\sigma_{k} & >\sigma_{d o}
\end{aligned}
$$

In this study, the standard value of $1 / 8$ was used for $\sigma_{d o}$ in all flow solutions. Acceptable ranges for $\sigma_{w}$ and $\sigma_{k}$ were established from inequalities (22) and (23) and from Figs. 4.16 and 4.17 in Ref. 13. In private communication with the first author, ${ }^{14}$ Wilcox stated that the standard value of $C_{\text {lim }}$ was chosen mainly for applications with Mach number greater than two. For the transonic range, Wilcox recommended a value of $C_{\text {lim }}$ closer to one.

In total, six closure coefficients were varied in the UQ analysis for W2006. A summary of the W2006 closure coefficients to be varied and their epistemic intervals are included in Table 2 . 
Table 2. W2006 Closure Coefficients and Associated Epistemic Intervals.

\begin{tabular}{cccc}
\hline Coefficient & Standard Value & Lower Bound & Upper Bound \\
\hline$\beta^{*}$ & 0.09 & 0.0784 & 0.1024 \\
$C_{\text {lim }}$ & 0.875 & 0.75 & 1.0 \\
$\kappa$ & 0.40 & 0.38 & 0.42 \\
$\beta^{*} / \beta_{0}$ & 1.2712 & 1.19 & 1.31 \\
$\sigma_{w}$ & 0.5 & 0.5 & 0.7 \\
$\sigma_{k}$ & 0.6 & 0.5 & 0.6 \\
\hline
\end{tabular}

\section{III.C. Menter Shear-Stress Transport Two-Equation Model (SST)}

Menter's SST model uses blending functions to combine the $k-\omega$ and $k-\epsilon$ models. ${ }^{3}$ The objective is to use the $k-\omega$ model in the near wall region where it is most accurate, and to take advantage of the freestream independence of the $k-\epsilon$ model in the outer part of the boundary layer. The model is given by

$$
\begin{aligned}
& \frac{\partial(\rho k)}{\partial t}+\frac{\partial\left(\rho u_{j} k\right)}{\partial x_{j}}=P-\beta^{*} \rho \omega k+\frac{\partial}{\partial x_{j}}\left[\left(\mu+\sigma_{k} \mu_{t}\right) \frac{\partial k}{\partial x_{j}}\right] \\
& \frac{\partial(\rho \omega)}{\partial t}+\frac{\partial\left(\rho u_{j} \omega\right)}{\partial x_{j}}=\frac{\gamma}{\nu_{t}} P-\beta \rho \omega^{2}+\frac{\partial}{\partial x_{j}}\left[\left(\mu+\sigma_{w} \mu_{t}\right) \frac{\partial \omega}{\partial x_{j}}\right]+2\left(1-F_{1}\right) \frac{\rho \sigma_{w 2}}{\omega} \frac{\partial k}{\partial x_{j}} \frac{\partial \omega}{\partial x_{j}}
\end{aligned}
$$

The full formulation of the model is given by Menter. ${ }^{3}$ The closure coefficients and their suggested values are given in Table 3 . The closure coefficients also include

$$
\begin{aligned}
\gamma_{1} & =\frac{\beta_{1}}{\beta^{*}}-\sigma_{w 1} \frac{\kappa^{2}}{\sqrt{\beta^{*}}} \\
\gamma_{2} & =\frac{\beta_{2}}{\beta^{*}}-\sigma_{w 2} \frac{\kappa^{2}}{\sqrt{\beta^{*}}}
\end{aligned}
$$

As in the W2006 model, $P, \tau_{i j}$, and $S_{i j}$ are given by:

$$
\begin{aligned}
P & =\tau_{i j} \frac{\partial u_{i}}{\partial x_{j}} \\
\tau_{i j} & =\mu_{t}\left(2 S_{i j}-\frac{2}{3} \frac{\partial u_{k}}{\partial x_{k}} \delta_{i j}\right)-\frac{2}{3} \rho k \delta_{i j} \\
S_{i j} & =\frac{1}{2}\left(\frac{\partial u_{i}}{\partial x_{j}}+\frac{\partial u_{j}}{\partial x_{i}}\right)
\end{aligned}
$$

but the turbulent eddy viscosity is computed from:

$$
\mu_{t}=\frac{\rho a_{1} k}{\max \left(a_{1} \omega, \Omega F_{2}\right)}
$$

Each of the constants is a blend of an inner (1) and outer (2) constant, blended via

$$
\phi=F_{1} \phi_{1}+\left(1-F_{1}\right) \phi_{2}
$$

where $\phi_{1}$ represents constant 1 and $\phi_{2}$ represents constant 2. Additional functions are given by

$$
\begin{array}{rlrl}
F_{1} & =\tanh \left(\arg _{1}^{4}\right), & \arg g_{1} & =\min \left[\max \left(\frac{\sqrt{k}}{\beta^{*} \omega d}, \frac{500 \nu}{d^{2} \omega}\right), \frac{4 \rho \sigma_{\omega 2} k}{C D_{k \omega} d^{2}}\right] \\
\nu_{t}=\frac{\mu_{t}}{\rho}, & C D_{k \omega} & =\max \left(2 \rho \sigma_{\omega 2} \frac{1}{\omega} \frac{\partial k}{\partial x_{j}} \frac{\partial \omega}{\partial x_{j}}, 10^{-20}\right) \\
F_{2} & =\tanh \left(\arg _{2}^{2}\right), & \arg _{2} & =\max \left(2 \frac{\sqrt{k}}{\beta^{*} \omega d}, \frac{500 \nu}{d^{2} \omega}\right) \\
\Omega & =\sqrt{2 W_{i j} W_{i j}}, & W_{i j} & =\frac{1}{2}\left(\frac{\partial u_{i}}{\partial x_{j}}-\frac{\partial u_{j}}{\partial x_{i}}\right)
\end{array}
$$


Table 3. SST Closure Coefficients and Associated Epistemic Intervals.

\begin{tabular}{cccc}
\hline Coefficient & Standard Value & Lower Bound & Upper Bound \\
\hline$\sigma_{k 1}$ & 0.85 & 0.7 & 1.0 \\
$\sigma_{k 2}$ & 1.0 & 0.8 & 1.2 \\
$\sigma_{w 1}$ & 0.5 & 0.3 & 0.7 \\
$\sigma_{w 2}$ & 0.856 & 0.7 & 1.0 \\
$\beta^{*} / \beta_{1}$ & 1.20 & 1.19 & 1.31 \\
$\beta^{*} / \beta_{2}$ & 1.0870 & 1.05 & 1.45 \\
$\beta^{*}$ & 0.09 & 0.0784 & 0.1024 \\
$\kappa$ & 0.41 & 0.38 & 0.42 \\
$a_{1}$ & 0.31 & 0.31 & 0.40 \\
\hline
\end{tabular}

where $\rho$ is the density, $\nu_{t}$ is the turbulent kinematic viscosity, $\mu$ is the dynamic viscosity, $d$ is the distance from the field point to the nearest wall, and $\Omega$ is the vorticity magnitude.

Menter states that, "It has been the author's experience that small changes (5-10\%) in modeling constraints can lead to a significant improvement (or deterioration) of model predictions." ${ }^{3}$ Unfortunately, little more information is available regarding plausible domains for SST closure coefficients. Many of the SST closure coefficients are shared with W2006, and in these cases the same epistemic intervals from W2006 were employed for SST. Georgiadis and Yoder ${ }^{15}$ determined that $a_{1} \in[0.31,0.40]$. Menter agreed with this assessment in private communication with the first author, ${ }^{16}$ stating that "One can only increase $a_{1}$ decreasing it interferes with the log layer calibration."

In total, nine closure coefficients were varied in the UQ analysis for SST. A summary of the SST closure coefficients to be varied and their epistemic intervals is included in Table 3.

\section{Uncertainty Quantification Approach}

Uncertainties in computational models fall into two categories: aleatory and epistemic. Aleatory uncertainties represent inherent variations in a system, whereas epistemic uncertainties arise due to lack of knowledge. (For example, the outcome of a coin flip is aleatory because it is due to inherent chance; however if a coin is placed on a table and covered up, the uncertainty in heads/tails is epistemic because it is due to a lack of knowledge.) In this work, all closure coefficients are treated as epistemic uncertain variables due to the lack of a complete physical understanding of turbulence.

Rather than resorting to Monte Carlo simulations for uncertainty quantification, stochastic expansions based on Point-Collocation Non-Intrusive Polynomial Chaos (NIPC) were employed to reduce computational expense. ${ }^{5}$ The strategy of Point-Collocation NIPC is to create a surrogate model via least squares approach (i.e., polynomial response surface) by using the CFD output obtained at a number of Latin Hypercube sample points for the propagation of uncertainty. An explanation of Point-Collocation NIPC given by West et al. ${ }^{17}$ follows. With the polynomial chaos approach, a stochastic response function $\alpha^{*}$ (e.g., drag coefficient, pressure or skin friction coefficient at a given point in the flow field) can be decomposed into separable deterministic and stochastic components within a series expansion:

$$
\alpha^{*}(\boldsymbol{x}, \boldsymbol{\xi}) \approx \sum_{i=0}^{P} \alpha_{i}(\boldsymbol{x}) \Psi_{i}(\boldsymbol{\xi})
$$

where $\alpha_{i}$ is the deterministic component and $\Psi_{i}$ is the random variable basis functions corresponding to the $i^{\text {th }}$ mode. $\alpha^{*}$ is assumed to be a function of a deterministic vector $\boldsymbol{x}$, which includes the spatial coordinates and deterministic parameters of the problem, and of the $n$-dimensional standard random variable vector $\boldsymbol{\xi}$. In theory, the series in Eq. (34) is infinite, but for practical implementation of the polynomial chaos expansions it is truncated and a discrete sum is taken over a number of output modes, $(P+1)$. Further details on polynomial chaos theory are given by Ghanem ${ }^{18}$ and Eldred. ${ }^{19}$

There are three parameters that determine the number of samples required to generate the response surface: the number of uncertain variables, $n$; the order of the response surface polynomial, $p$; and the 
oversampling ratio, $n_{p}$. The total number of samples, $N_{s}$, is then given by

$$
N_{s}=n_{p} \cdot(P+1)=n_{p}\left[\frac{(n+p) !}{n ! p !}\right]
$$

The Point-Collocation NIPC method starts with replacing a stochastic response or random function with its polynomial chaos expansion in Eq. (34). Then, $N_{s}$ vectors are chosen in random space and the deterministic code (the CFD flow solver in this case) is evaluated at these points; this is the left-hand side of Eq. (34). Finally a linear system of $N_{s}$ equations is formulated and solved for the spectral modes of the random variables. This system is given by:

$$
\left(\begin{array}{c}
\alpha^{*}\left(\boldsymbol{x}, \boldsymbol{\xi}_{0}\right) \\
\alpha^{*}\left(\boldsymbol{x}, \boldsymbol{\xi}_{1}\right) \\
\vdots \\
\alpha^{*}\left(\boldsymbol{x}, \boldsymbol{\xi}_{\left(N_{s}-1\right)}\right)
\end{array}\right)=\left(\begin{array}{cccc}
\Psi_{0}\left(\boldsymbol{\xi}_{0}\right) & \Psi_{1}\left(\boldsymbol{\xi}_{0}\right) & \ldots & \Psi_{P}\left(\boldsymbol{\xi}_{0}\right) \\
\Psi_{0}\left(\boldsymbol{\xi}_{1}\right) & \Psi_{1}\left(\boldsymbol{\xi}_{1}\right) & \ldots & \Psi_{P}\left(\boldsymbol{\xi}_{1}\right) \\
\vdots & \vdots & \ddots & \vdots \\
\Psi_{0}\left(\boldsymbol{\xi}_{\left(N_{s}-1\right)}\right) & \Psi_{1}\left(\boldsymbol{\xi}_{\left(N_{s}-1\right)}\right) & \ldots & \Psi_{P}\left(\boldsymbol{\xi}_{\left(N_{s}-1\right)}\right)
\end{array}\right)\left(\begin{array}{c}
\alpha_{0} \\
\alpha_{1} \\
\vdots \\
\alpha_{P}
\end{array}\right)
$$

An oversampling ratio of 1.0 yields the minimum number of samples required to produce a response surface. Hosder et al. ${ }^{20}$ demonstrated that an oversampling ratio of $n_{p}=2.0$ gives a better approximation to the statistics at each polynomial degree they considered. For this reason, $n_{p}=2.0$ was used for all of the UQ analyses in this work. Given $n_{p}>1.0$, Eq. (36) is overdetermined and can be solved using a least squares approach. In the current work, a polynomial order of two $(p=2)$ was used for all UQ analyses.

Sobol indices (global nonlinear sensitivity indices) were used to rank the relative contributions of each closure coefficient to the total uncertainty in the output quantities of interest. Sobol indices can be derived via Sobol Decomposition, which is a variance-based global sensitivity analysis method. This derivation utilizes the polynomial chaos expansion coefficients calculated in Eq. (36), and is included in Appendix A.

Due to the bounded nature of epistemic input uncertainties, Legendre polynomials are used in this study as the basis functions. Although stochastic response surfaces created with the NIPC approach allow the calculation of confidence intervals along with various statistics of the output for probabilistic (aleatory) input, in this study only the maximum and the minimum of the response will be calculated from the response surface to determine the epistemic interval for each uncertain output.

Initially all of the closure coefficients in Tables 1, 2, and 3 were used in the uncertainty quantification analyses. The results of these analysis are presented in the next section. The closure coefficients that contribute most to the uncertain outputs in the original analyses are then used as the uncertain variables in a second set of reduced-dimensionality analyses (RDA). The objective of the second set of analyses is to obtain more accurate response surfaces and Sobol indices for the significant closure coefficients. A summary of the computational cost (i.e., number of CFD evaluations required) of the transonic bump analyses is included in Table 4, and a summary of the computational cost of the RAE 2822 analyses is included in Table 5 .

\section{Results}

The results of all uncertainty quantification analyses are presented in this section. The axisymmetric, transonic bump is discussed in Sections V.A and V.B; and the RAE 2822 transonic airfoil is discussed in Sections V.C and V.D. Finally, a comparison of the results from both CFD problems is discussed in Section V.E.

Table 4. Number of CFD Evaluations Required for Transonic Bump UQ Analyses.

\begin{tabular}{ccccccccc}
\hline & \multicolumn{4}{c}{ Original Analysis } & \multicolumn{4}{c}{$\mathrm{RDA}$} \\
Turbulence Model & $n$ & $p$ & $n_{p}$ & \multicolumn{1}{c}{$N_{s}$} & $n$ & $p$ & $n_{p}$ & $N_{s}$ \\
\hline SA & 11 & 2 & 2 & 156 & 6 & 2 & 2 & 56 \\
W2006 & 6 & 2 & 2 & 56 & 3 & 2 & 2 & 20 \\
SST & 9 & 2 & 2 & 110 & 5 & 2 & 2 & 42 \\
\hline
\end{tabular}


Table 5. Number of CFD Evaluations Required for RAE 2822 UQ Analyses.

\begin{tabular}{ccccccccc}
\hline & \multicolumn{4}{c}{ Original Analysis } & \multicolumn{4}{c}{ RDA } \\
Turbulence Model & $n$ & $p$ & $n_{p}$ & \multicolumn{1}{c}{$N_{s}$} & $n$ & $p$ & $n_{p}$ & $N_{s}$ \\
\hline SA & 11 & 2 & 2 & 156 & 7 & 2 & 2 & 72 \\
W2006 & 6 & 2 & 2 & 56 & 3 & 2 & 2 & 20 \\
SST & 9 & 2 & 2 & 110 & 5 & 2 & 2 & 42 \\
\hline
\end{tabular}

\section{V.A. Axisymmetric Transonic Bump - Original Analyses}

The purpose of the original uncertainty quantification analyses was to identify the closure coefficients that contribute significantly to uncertainty in the output quantities of interest. These coefficients were later used in reduced-dimensionality analyses. For the transonic bump, the output quantities of interest include drag coefficient $\left(C_{D}\right)$; pressure and skin friction coefficient distributions $\left(C_{p}\right.$ and $\left.C_{f}\right)$; and separation bubble size.

Sobol indices were used to rank the relative contributions of each closure coefficient to the overall uncertainty in $C_{D}$. Coefficients with higher Sobol indices contribute more to the uncertainty than coefficients with lower Sobol indices. The Sobol indices of the closure coefficients for each turbulence model for $C_{D}$ are presented in Table 6, where they are ranked from highest to lowest. The largest contributors to uncertainty in each model are typed in blue. Coefficients with Sobol indices of less than $3.0 \times 10^{-2}$ were not considered to be significant. The closure coefficients retained for each turbulence model with this approach account for at least $95 \%$ of the total uncertainty in the output quantity of interest (Table 6).

Table 6. Sobol Indices of Closure Coefficients for $C_{D}$.

\begin{tabular}{cccccc}
\hline \multicolumn{2}{c}{ SA } & \multicolumn{2}{c}{ W2006 } & \multicolumn{2}{c}{ SST } \\
Coefficient & Sobol Index & Coefficient & Sobol Index & Coefficient & Sobol Index \\
\hline$\kappa$ & $8.50 \times 10^{-1}$ & $\sigma_{w}$ & $7.75 \times 10^{-1}$ & $\beta^{*}$ & $8.03 \times 10^{-1}$ \\
$\sigma$ & $5.99 \times 10^{-2}$ & $C_{\text {lim }}$ & $1.42 \times 10^{-1}$ & $\sigma_{w 1}$ & $9.24 \times 10^{-2}$ \\
$c_{v 1}$ & $5.38 \times 10^{-2}$ & $\beta^{*}$ & $1.26 \times 10^{-1}$ & $\beta^{*} / \beta_{1}$ & $7.79 \times 10^{-2}$ \\
$c_{w 2}$ & $2.59 \times 10^{-2}$ & $\sigma_{k}$ & $2.12 \times 10^{-2}$ & $\beta^{*} / \beta_{2}$ & $3.54 \times 10^{-2}$ \\
$c_{b 1}$ & $1.11 \times 10^{-2}$ & $\kappa$ & $1.99 \times 10^{-2}$ & $\sigma_{w 2}$ & $1.85 \times 10^{-3}$ \\
$c_{b 2}$ & $4.66 \times 10^{-4}$ & $\beta^{*} / \beta_{0}$ & $7.46 \times 10^{-3}$ & $\sigma_{k 2}$ & $1.21 \times 10^{-3}$ \\
$c_{w 3}$ & $5.33 \times 10^{-5}$ & & & $a_{1}$ & $8.25 \times 10^{-4}$ \\
$c_{t 3}$ & $9.71 \times 10^{-6}$ & & & $\sigma_{k 1}$ & $4.91 \times 10^{-4}$ \\
$c_{t 1}$ & $8.91 \times 10^{-6}$ & & & $\kappa$ & $1.24 \times 10^{-4}$ \\
$c_{t 4}$ & $8.57 \times 10^{-6}$ & & & & \\
$c_{t 2}$ & $5.13 \times 10^{-6}$ & & & & \\
\hline
\end{tabular}

The largest contributors to uncertainty in $C_{D}$ for the SA model are $\kappa, \sigma$, and $c_{v 1}$. The largest contributors for W2006 are $\sigma_{w}, C_{l i m}$, and $\beta^{*}$. The largest contributors for SST are $\beta^{*}, \sigma_{w 1}, \beta^{*} / \beta_{1}$, and $\beta^{*} / \beta_{2}$. Note that $\beta^{*}$ is shared by both the W2006 and SST models. Also note that $\sigma_{w 1}$ in the SST model is the equivalent of $\sigma_{w}$ in the W2006 model. Von Kármán's constant $(\kappa)$ is included in all three models, but it is only significant to uncertainty in $C_{D}$ for the SA model.

Response surfaces for pressure coefficient $\left(C_{p}\right)$ and skin friction coefficient $\left(C_{f}\right)$ were generated at each axial location across the bump. Sobol indices for these coefficients were also calculated at each axial location. Plots of the Sobol indices for $C_{p}$ and $C_{f}$ versus $x$ were generated so that the closure coefficients significant to these quantities at points in the flow could be identified. These plots are included in Fig. 5. For clarity, only the Sobol indices of coefficients with significant contributions to uncertainty in $C_{p}$ are shown in the plots.

The largest contributors to uncertainty in $C_{p}$ and $C_{f}$ for the SA model are $\sigma, \kappa, c_{v 1}, c_{w 3}, c_{b 1}$, and $c_{w 2}$. The largest contributors for the W2006 model are $\beta^{*}, C_{\text {lim }}$, and $\sigma_{w}$. The largest contributors for the SST model are $\sigma_{w 1}, \beta^{*} / \beta_{1}, \beta^{*} / \beta_{2}, \beta^{*}$, and $a_{1}$.

The separation bubble size for each case of each model was taken as the difference between the $x$ coordinate of the zero-intersection points in the $C_{f}$ versus $x$ curves. Using the same process as before, 


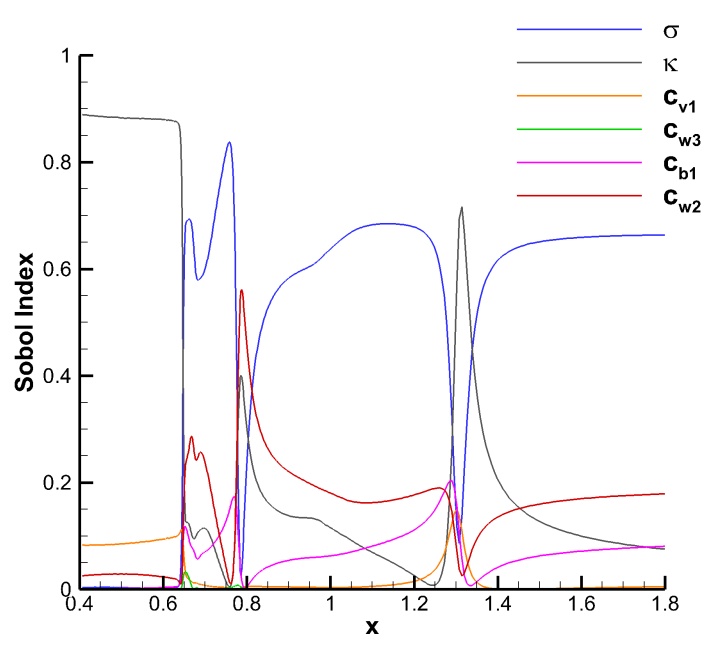

(a) Sobol indices for $\boldsymbol{C}_{\boldsymbol{p}}$ versus $\boldsymbol{x}$ (SA).

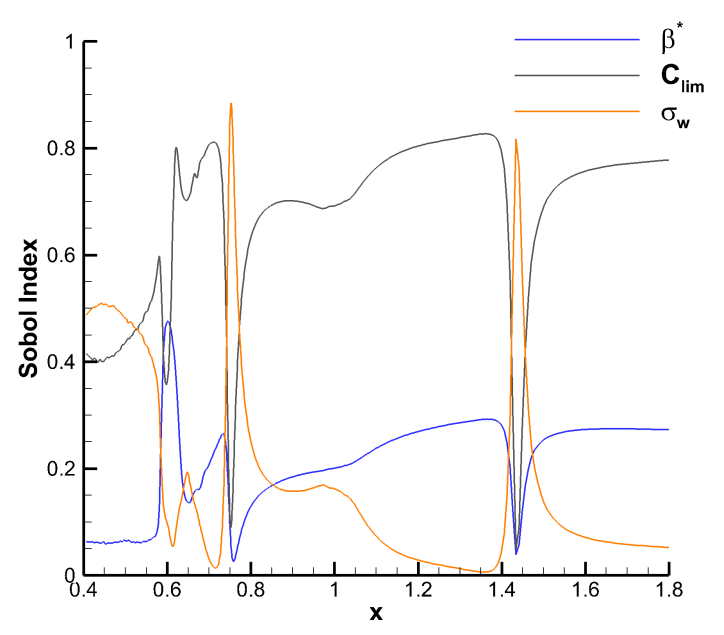

(c) Sobol indices for $\boldsymbol{C}_{\boldsymbol{p}}$ versus $\boldsymbol{x}$ (W2006).

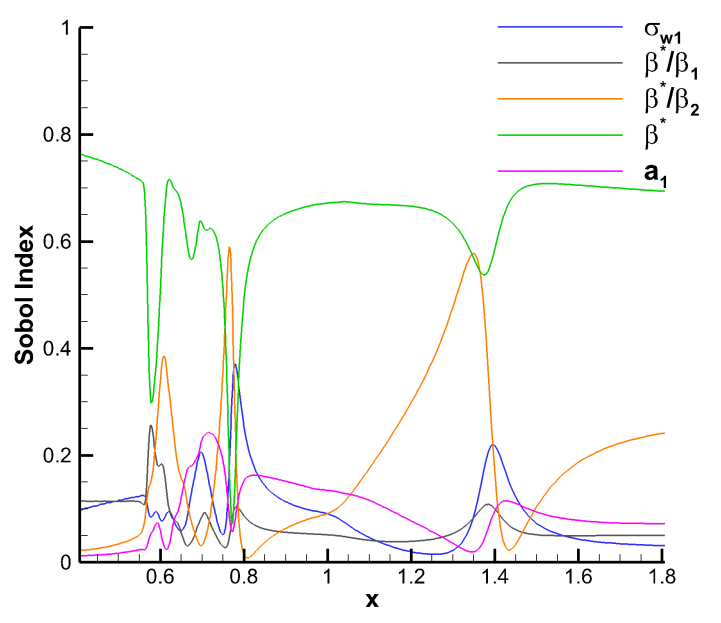

(e) Sobol indices for $\boldsymbol{C}_{\boldsymbol{p}}$ versus $\boldsymbol{x}$ (SST).

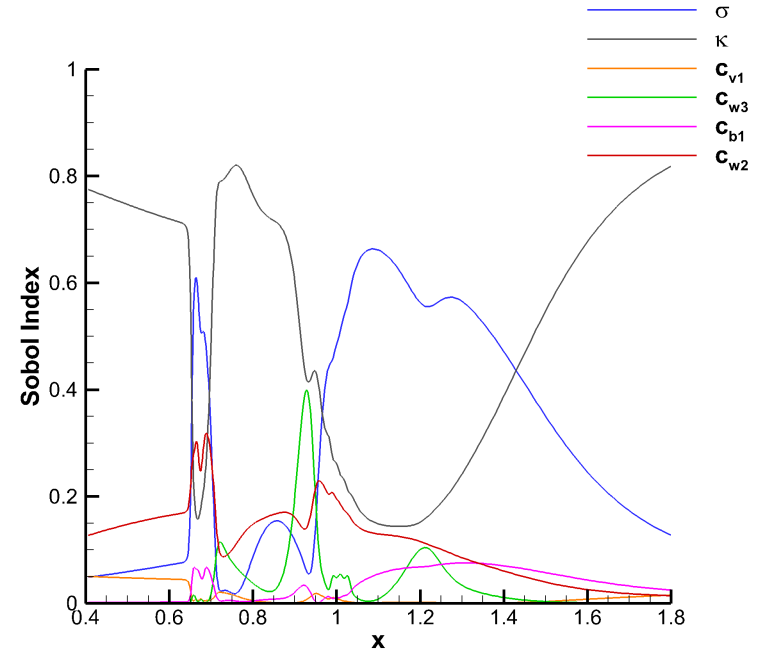

(b) Sobol indices for $\boldsymbol{C}_{\boldsymbol{f}}$ versus $\boldsymbol{x}(\mathrm{SA})$.

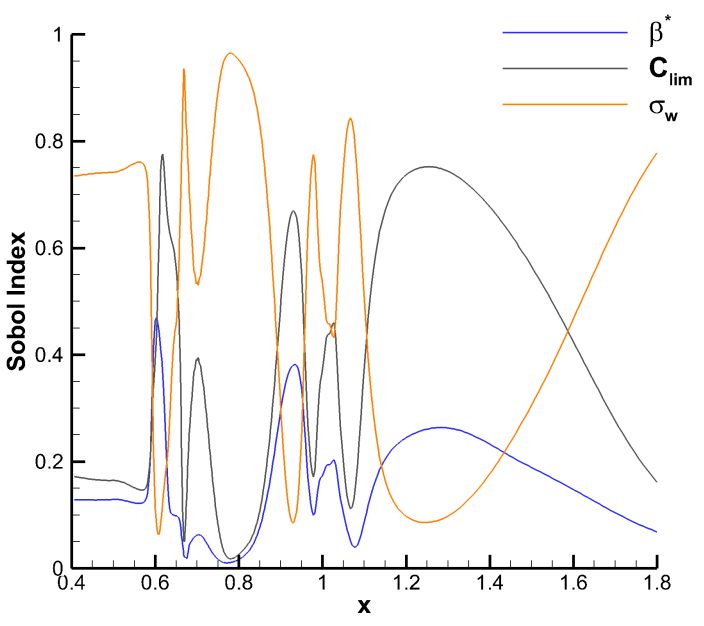

(d) Sobol indices for $\boldsymbol{C}_{\boldsymbol{f}}$ versus $\boldsymbol{x}$ (W2006).

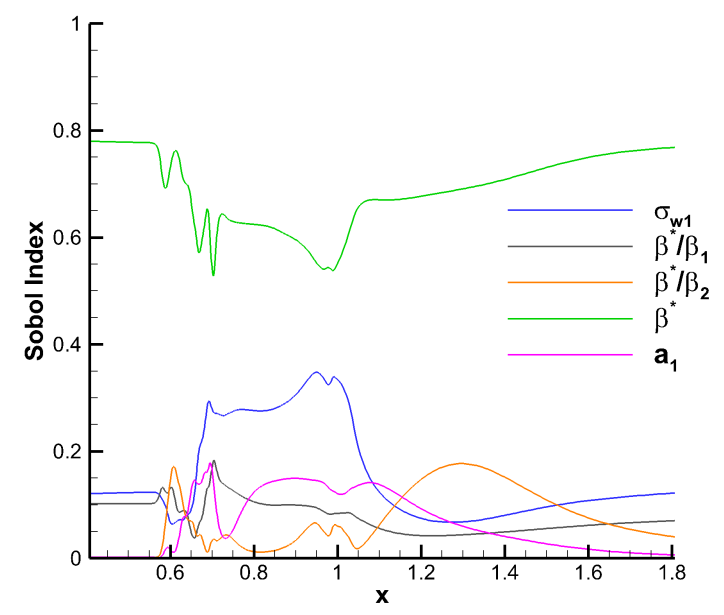

(f) Sobol indices for $\boldsymbol{C}_{\boldsymbol{f}}$ versus $\boldsymbol{x}$ (SST).

Figure 5. Sobol index distributions for transonic bump (Original Analyses). 
Table 7. Sobol Indices of Closure Coefficients for Separation Bubble Size.

\begin{tabular}{llllll}
\hline & \multicolumn{2}{c}{ SA } & \multicolumn{2}{c}{ W2006 } & \multicolumn{2}{c}{ SST } \\
Coefficient & Sobol Index & Coefficient & Sobol Index & Coefficient & Sobol Index \\
\hline$\sigma$ & $5.58 \times 10^{-1}$ & $C_{\text {lim }}$ & $7.26 \times 10^{-1}$ & $\beta^{*}$ & $6.49 \times 10^{-1}$ \\
$\kappa$ & $1.82 \times 10^{-1}$ & $\beta^{*}$ & $2.57 \times 10^{-1}$ & $\sigma_{w 1}$ & $1.15 \times 10^{-1}$ \\
$c_{w 2}$ & $1.54 \times 10^{-1}$ & $\sigma_{w}$ & $1.03 \times 10^{-1}$ & $a_{1}$ & $1.06 \times 10^{-1}$ \\
$c_{b 1}$ & $6.74 \times 10^{-2}$ & $\sigma_{k}$ & $1.46 \times 10^{-2}$ & $\beta^{*} / \beta_{2}$ & $9.48 \times 10^{-2}$ \\
$c_{w 3}$ & $3.71 \times 10^{-2}$ & $\kappa$ & $9.92 \times 10^{-3}$ & $\beta^{*} / \beta_{1}$ & $9.24 \times 10^{-2}$ \\
$c_{v 1}$ & $1.87 \times 10^{-3}$ & $\beta^{*} / \beta_{0}$ & $5.51 \times 10^{-3}$ & $\sigma_{k 2}$ & $2.09 \times 10^{-2}$ \\
$c_{t 3}$ & $6.76 \times 10^{-4}$ & & & $\kappa$ & $1.70 \times 10^{-2}$ \\
$c_{t 4}$ & $6.05 \times 10^{-4}$ & & & $\sigma_{w 2}$ & $1.36 \times 10^{-2}$ \\
$c_{t 2}$ & $5.98 \times 10^{-4}$ & & & $\sigma_{k 1}$ & $9.77 \times 10^{-3}$ \\
$c_{b 2}$ & $5.68 \times 10^{-4}$ & & & & \\
$c_{t 1}$ & $5.56 \times 10^{-4}$ & & & & \\
\hline
\end{tabular}

Table 8. Closure Coefficients with Significant Contributions to Transonic Bump.

\begin{tabular}{ccc}
\hline SA & W2006 & SST \\
\hline$\sigma$ & $\beta^{*}$ & $\sigma_{w 1}$ \\
$\kappa$ & $C_{l i m}$ & $\beta^{*} / \beta_{1}$ \\
$c_{v 1}$ & $\sigma_{w}$ & $\beta^{*} / \beta_{2}$ \\
$c_{w 2}$ & & $\beta^{*}$ \\
$c_{b 1}$ & & $a_{1}$ \\
$c_{w 3}$ & & \\
\hline
\end{tabular}

stochastic response surfaces were created for the separation bubble size for each model. The Sobol indices of the closure coefficients for separation bubble size are given in Table 7 .

The largest contributors to uncertainty in separation bubble size for the SA model are $\sigma, \kappa, c_{w 2}, c_{b 1}$, and $c_{w 3}$. The largest contributors for the W2006 model are $C_{l i m}, \beta^{*}$, and $\sigma_{w}$. The largest contributors for the SST model are $\beta^{*}, \sigma_{w 1}, a_{1}, \beta^{*} / \beta_{2}$, and $\beta^{*} / \beta_{1}$.

In sum, six closure coefficients were found to contribute significantly to at least one output quantity of interest for the SA model; three closure coefficients for the W2006 model; and five coefficients for the SST model. This information is summarized in Table 8.

\section{V.B. Axisymmetric Transonic Bump - Reduced Dimensionality Analyses}

To further increase the accuracy of the uncertainty analysis by creating the stochastic response surface with a smaller number of uncertain variables, a reduced dimensionality analysis (RDA) was performed for the axisymmetric transonic bump with each turbulence model by using only the significant closure coefficients found in Table 8.

A baseline case was run with the standard set of closure coefficients for each turbulence model. Stochastic polynomial response surfaces of $C_{D}$ were obtained with NIPC on the uncertain domain. The minimum and maximum values of drag coefficient $\left(C_{D}\right)$ for each model were calculated from these response surfaces. To verify the accuracy of each response surface, additional CFD solutions were generated by using the sets of closure coefficients that produced the minimum and maximum values of $C_{D}$ on the response surface; the $C_{D}$ comparison between the response surfaces and the CFD is summarized in Table 9 . In each case, the response surface is within $4.07 \%$ or less of the CFD. The difference values reported in Table 9 indicate an acceptable level of accuracy for epistemic uncertainty analyses.

In the experiment, Bachalo and Johnson estimated the separation and reattachment points to be $x=0.70$ and $x=1.1$ respectively. This yields a separation bubble size of $0.40(x / c)$ (based on the bump length of 
Table 9. Drag Coefficient Results, in Drag Counts (RDA).

\begin{tabular}{cccccccc}
\hline & Baseline $C_{D}$ & \multicolumn{3}{c}{ Min $C_{D}$} & \multicolumn{3}{c}{ Max $C_{D}$} \\
Model & CFD & Resp. Surf. & CFD & Difference & Resp. Surf. & CFD & Difference \\
\hline SA & 2.232 & 2.148 & 2.130 & $0.85 \%$ & 2.270 & 2.288 & $0.79 \%$ \\
W2006 & 2.226 & 2.116 & 2.098 & $0.86 \%$ & 2.243 & 2.224 & $0.85 \%$ \\
SST & 2.284 & 1.813 & 1.890 & $4.07 \%$ & 2.647 & 2.612 & $1.34 \%$ \\
\hline
\end{tabular}

Table 10. Separation Bubble Size Results (RDA).

\begin{tabular}{cccc}
\hline Model & $\begin{array}{c}\text { Baseline Size } \\
\text { CFD }\end{array}$ & $\begin{array}{c}\text { Min Size } \\
\text { Resp. Surf. }\end{array}$ & $\begin{array}{c}\text { Max Size } \\
\text { Resp. Surf. }\end{array}$ \\
\hline SA & 0.467 & 0.441 & 0.507 \\
W2006 & 0.535 & 0.522 & 0.672 \\
SST & 0.507 & 0.000 & 0.903 \\
\hline
\end{tabular}

Table 11. Sobol Indices of Closure Coefficients for $C_{D}$ (RDA).

\begin{tabular}{cccccc}
\hline \multicolumn{2}{c}{ SA } & \multicolumn{2}{c}{ W2006 } & \multicolumn{2}{c}{ SST } \\
Coefficient & Sobol Index & Coefficient & Sobol Index & Coefficient & Sobol Index \\
\hline$\kappa$ & $8.51 \times 10^{-1}$ & $\sigma_{w}$ & $7.26 \times 10^{-1}$ & $\beta^{*}$ & $7.95 \times 10^{-1}$ \\
$\sigma$ & $6.08 \times 10^{-2}$ & $\beta^{*}$ & $2.07 \times 10^{-1}$ & $\sigma_{w 1}$ & $9.19 \times 10^{-2}$ \\
$c_{v 1}$ & $5.35 \times 10^{-2}$ & $C_{l i m}$ & $1.89 \times 10^{-1}$ & $\beta^{*} / \beta_{1}$ & $7.96 \times 10^{-2}$ \\
$c_{w 2}$ & $2.56 \times 10^{-2}$ & & & $\beta^{*} / \beta_{2}$ & $4.86 \times 10^{-2}$ \\
$c_{b 1}$ & $1.08 \times 10^{-2}$ & & & $a_{1}$ & $3.01 \times 10^{-3}$ \\
$c_{w 3}$ & $3.17 \times 10^{-5}$ & & & & \\
\hline
\end{tabular}

$c=1)$. The separation bubble size results for the UQ analyses are included in Table 10. Originally, the SST response surface for separation bubble size yielded a negative minimum bubble size. This was due to the fact that for certain samples there was a very small, or even no separation region, yielding negative values for the response. Since this is not physical, the minimum value is set to zero.

The Sobol indices for $C_{D}$ and separation bubble size agree well with the Sobol indices in the original analyses. These values are included in Tables 11 and 12. The ranking of importance is mostly the same between the original and reduced-dimensionality analyses. Even when the ranking of importance is not the same, the actual numeric values of the Sobol indices in the reduced-dimensionality analyses are still reasonably close to their corresponding values in the original analyses.

Response surfaces for pressure coefficient $\left(C_{p}\right)$ and skin friction coefficient $\left(C_{f}\right)$ were generated at each axial location across the bump. Sobol indices for these coefficients were also calculated at each axial location. The minimum and maximum possible values of $C_{p}$ and $C_{f}$ were calculated at each axial location from the response surfaces.

Plots of $C_{p}$ versus $x$ were generated for all of the cases used in the UQ analysis, for each turbulence model. This was done to verify the physical feasibility of the solutions. Each plot contains data for the baseline case; UQ training cases (green lines); minimum and maximum $C_{p}$ bounds; and experimental data from Bachalo and Johnson. ${ }^{4}$ The authors would like to emphasize that Bachalo and Johnson did not report any experimental uncertainty in their results, so the experimental data in these figures should be considered as reference only. A proper comparison with experiment should take into account the uncertainty both in the numerical model and experimental data, and the difference should be quantified with appropriate metrics for the validation of the numerical model.

The $C_{p}$ versus $x$ plots are included in Figs. 6a, 6c, and 6e. The Sobol indices for $C_{p}$ versus $x$ are included in Figs. 6b, 6d, and 6f. For clarity, only the Sobol indices of coefficients with significant contributions to uncertainty in $C_{p}$ are shown in the figures. For each model, additional cases were run at the corners of 
Table 12. Sobol Indices of Closure Coefficients for Separation Bubble Size (RDA).

\begin{tabular}{cccccc}
\hline \multicolumn{2}{c}{ SA } & \multicolumn{2}{c}{ W2006 } & \multicolumn{2}{c}{ SST } \\
Coefficient & Sobol Index & Coefficient & Sobol Index & Coefficient & Sobol Index \\
\hline$\sigma$ & $5.79 \times 10^{-1}$ & $C_{l i m}$ & $7.49 \times 10^{-1}$ & $\beta^{*}$ & $6.27 \times 10^{-1}$ \\
$\kappa$ & $1.84 \times 10^{-1}$ & $\beta^{*}$ & $2.71 \times 10^{-1}$ & $\beta^{*} / \beta_{1}$ & $1.86 \times 10^{-1}$ \\
$c_{w 2}$ & $1.45 \times 10^{-1}$ & $\sigma_{w}$ & $7.63 \times 10^{-2}$ & $\sigma_{w 1}$ & $1.37 \times 10^{-1}$ \\
$c_{b 1}$ & $5.91 \times 10^{-2}$ & & & $\beta^{*} / \beta_{2}$ & $1.24 \times 10^{-1}$ \\
$c_{w 3}$ & $3.27 \times 10^{-2}$ & & & $a_{1}$ & $7.99 \times 10^{-2}$ \\
$c_{v 1}$ & $3.65 \times 10^{-3}$ & & & & \\
\hline
\end{tabular}

the hypercube defined by the epistemic interval bounds of only the most significant closure coefficients; this was done to eliminate the effect of discontinuities in the response surfaces near the shock and to determine the epistemic uncertainty interval for the output in the shock region only. The $C_{p}$ minimum and maximum curves in Figs. 6a, 6c, and 6e are piece-wise combinations of the NIPC response surfaces and these hypercube cases in the shock region.

Note that each of the minimum and maximum $C_{p}$ curves may not in general correspond to one flow solution each, because each point along each curve is calculated from either the response surface generated by using the $C_{p}$ values of each UQ case at that specific $x$-location, or by one of the hypercube corner cases. While there exist flow solutions that pass through or very near to the minimum and maximum $C_{p}$ curves at each point, there is no flow solution that passes through all of the minimum or all of the maximum points.

Plots of skin friction coefficient at each axial location were created in a similar fashion as the plots of pressure coefficient. Plots of $C_{f}$ versus $x$ for each UQ case are included in Figs. 7a, 7c, and 7e. As before, the minimum and maximum $C_{f}$ curves in these figures are piece-wise combinations of the NIPC response surfaces at each axial location and hypercube corner samples in the shock region. The vertical lines in the plots are the separation and reattachment point locations reported by Bachalo and Johnson. ${ }^{4}$ The Sobol indices for $C_{f}$ at each axial location are shown in Figs. 7b, 7d, and $7 \mathrm{f}$.

Based on the reduced-dimensionality results, all of the coefficients found to be significant to the axisymmetric transonic bump in Section V.A are indeed significant to their respective turbulence models, even in the absence of possible numerical "noise" caused by the insignificant coefficients in the original analyses. Of the three turbulence models, the SST model is most sensitive to changes in its closure coefficients; this is evidenced by large variations in the model's values for $C_{D}$ and separation bubble size, as well as the large solution bands for $C_{p}$ and $C_{f}$. The SA and W2006 models are roughly equally sensitive to changes in their closure coefficients. All of the models are most sensitive to changes in their closure coefficients in the shock and separated regions of the flow, particularly so in the latter region. None of the models exhibit large uncertainties in $C_{p}$ upstream of the shock or downstream of the reattachment point, however the uncertainty in $C_{f}$ remains large in these regions.

\section{V.C. RAE 2822 Airfoil - Original Analyses}

As with the original analyses of the transonic bump, the purpose of the original analyses of the RAE 2822 airfoil was to identify the closure coefficients that contribute significantly to uncertainty in the output quantities of interest. For the RAE 2822, the output quantities of interest include the lift coefficient $\left(C_{L}\right)$, drag coefficient due to pressure $\left(C_{D_{\text {press }}}\right)$, drag coefficient due to skin friction $\left(C_{D_{\text {sf }}}\right)$, and pressure coefficient distribution $\left(C_{p}\right)$. The skin friction coefficient distribution was not investigated for the RAE 2822 because no separation bubble was observed in the baseline case solutions.

In the same manner as for the transonic bump, Sobol indices were calculated for the output quantities of interest of the RAE 2822. The results for $C_{L}, C_{D_{\text {press }}}$, and $C_{D_{\text {sf }}}$ are included in Tables 13,14 , and 15 respectively.

For the SA model, the largest contributors to uncertainty in $C_{L}$ are $\kappa, c_{t 4}, c_{t 3}, c_{v 1}$, and $c_{b 1}$. The largest contributors to uncertainty in $C_{D_{\text {press }}}$ are $\sigma, c_{w 2}, c_{b 1}$, and $c_{t 4}$; and the largest contributors to uncertainty in $C_{D_{\text {sf }}}$ are $\kappa, \sigma, c_{w 2}, c_{t 4}, c_{t 3}$, and $c_{v 1}$.

For the W2006 model, the largest contributors to uncertainty in all three integrated quantities are $C_{l i m}$, 


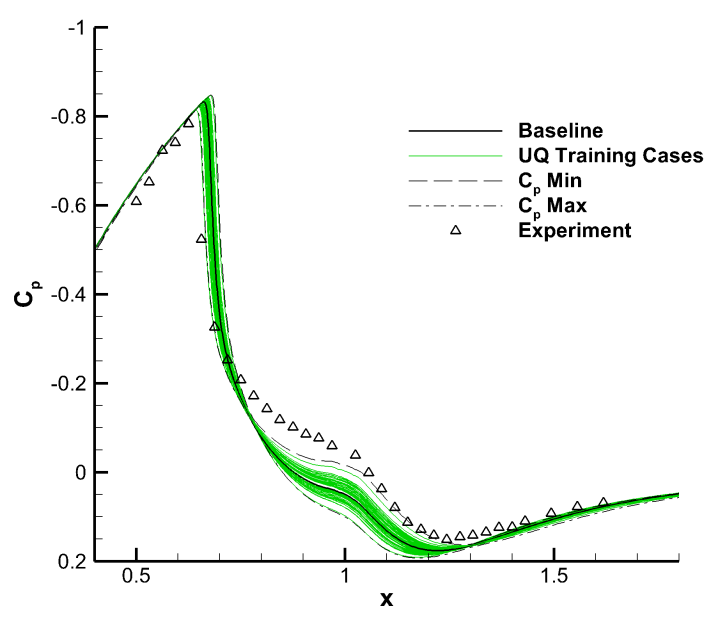

(a) $\boldsymbol{C}_{\boldsymbol{p}}$ versus $\boldsymbol{x}(\mathrm{SA})$.

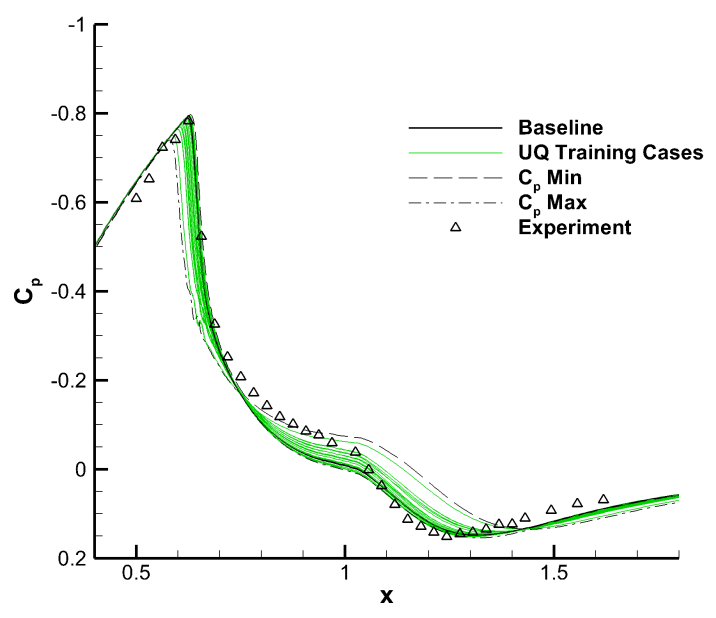

(c) $\boldsymbol{C}_{\boldsymbol{p}}$ versus $\boldsymbol{x}$ (W2006).

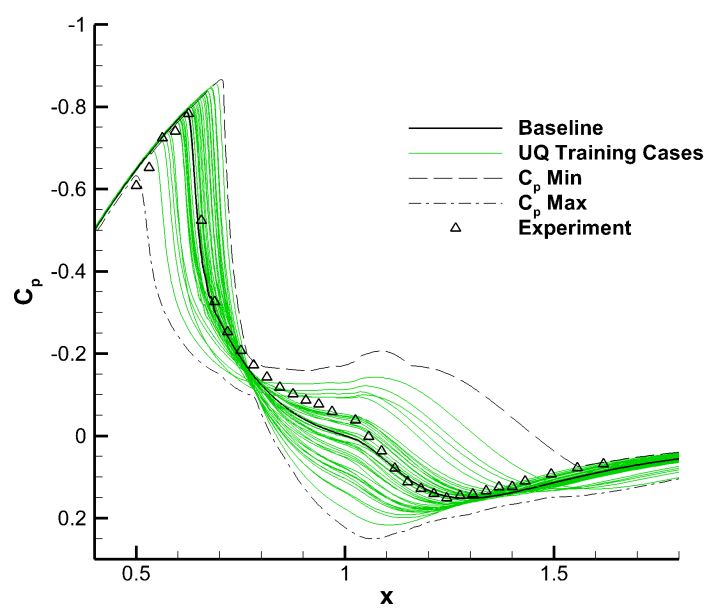

(e) $\boldsymbol{C}_{\boldsymbol{p}}$ versus $\boldsymbol{x}$ (SST).

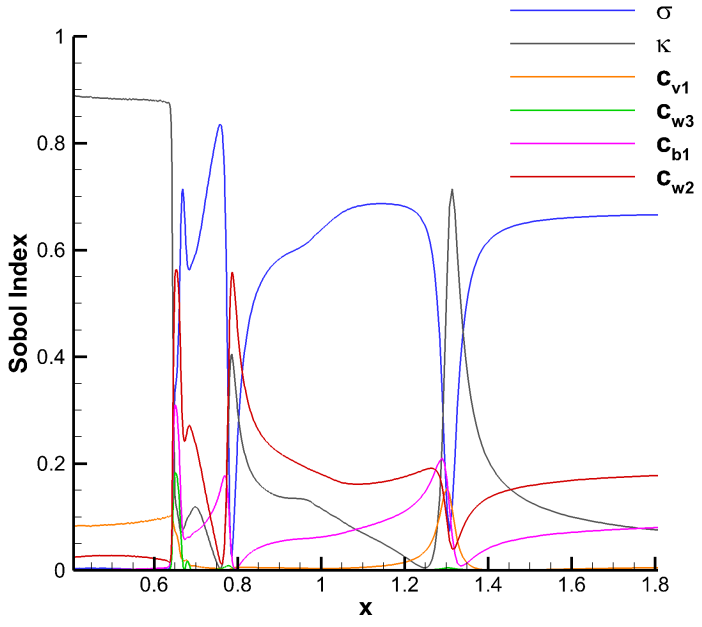

(b) Sobol indices for $\boldsymbol{C}_{\boldsymbol{p}}$ versus $\boldsymbol{x}(\mathrm{SA})$.

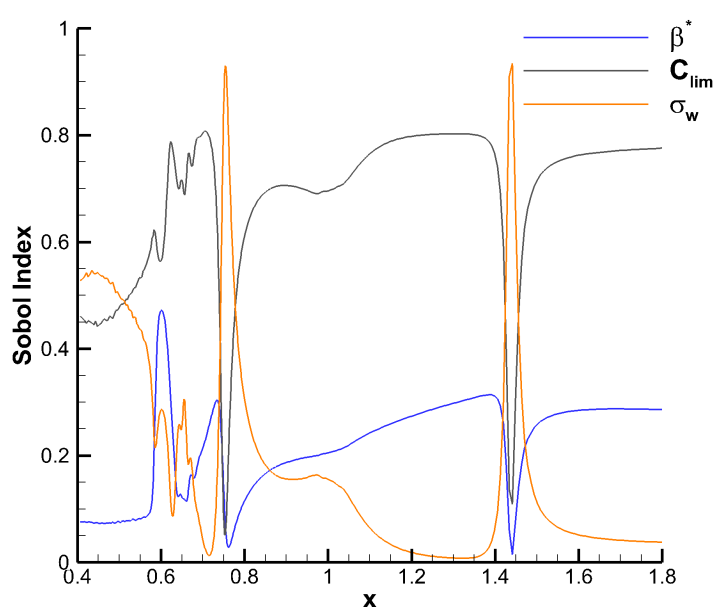

(d) Sobol indices for $\boldsymbol{C}_{\boldsymbol{p}}$ versus $\boldsymbol{x}$ (W2006).

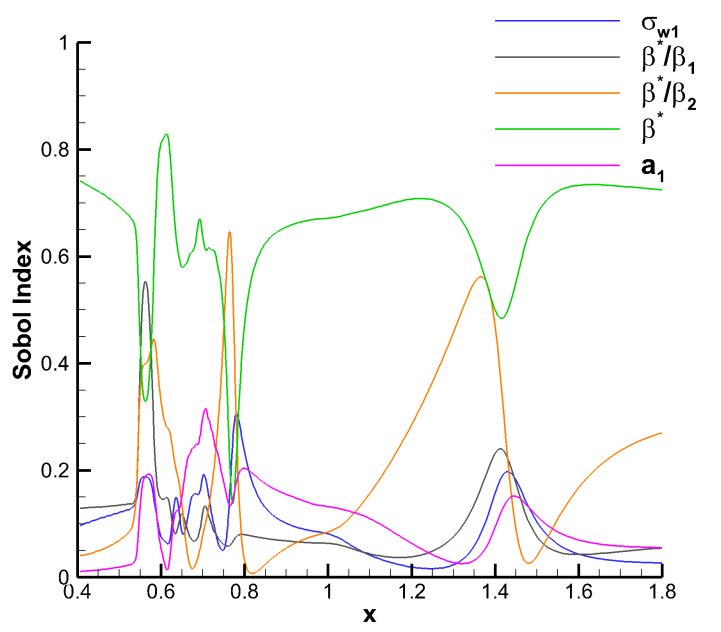

(f) Sobol indices for $\boldsymbol{C}_{\boldsymbol{p}}$ versus $\boldsymbol{x}$ (SST).

Figure 6. Pressure coefficient results for transonic bump.

\section{6 of 26}




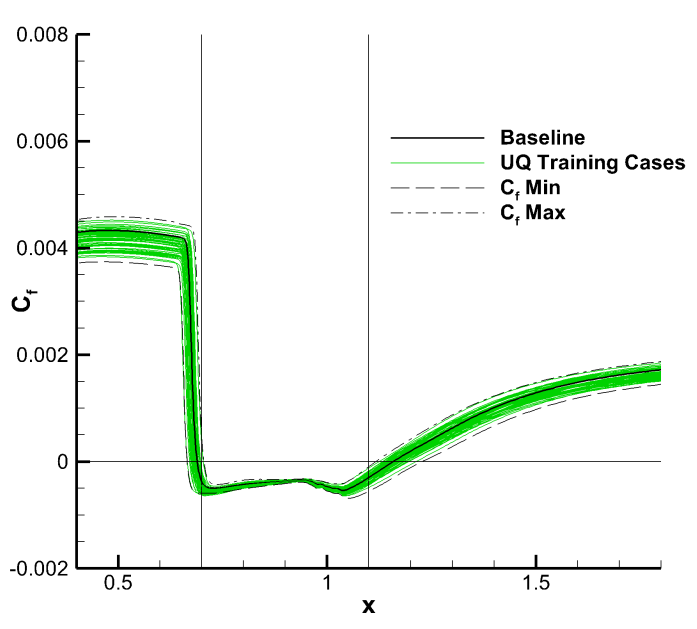

(a) $\boldsymbol{C}_{\boldsymbol{f}}$ versus $\boldsymbol{x}$ (SA).

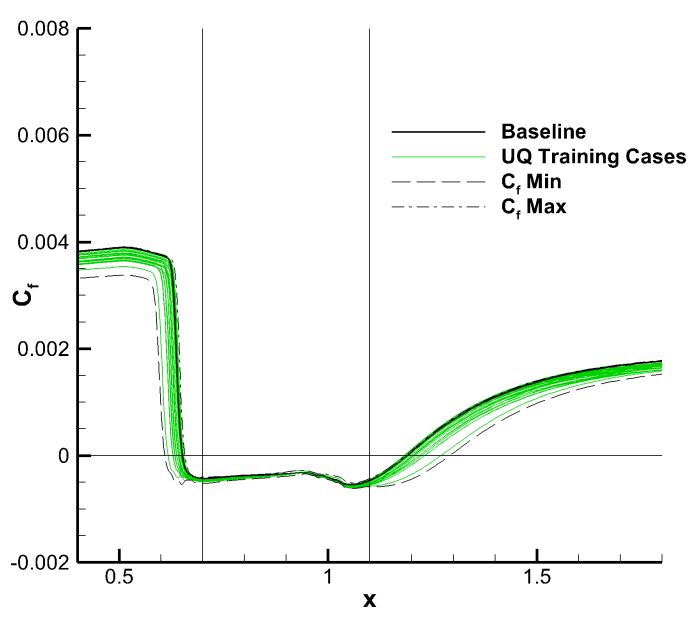

(c) $\boldsymbol{C}_{\boldsymbol{f}}$ versus $\boldsymbol{x}(\mathrm{W} 2006)$.

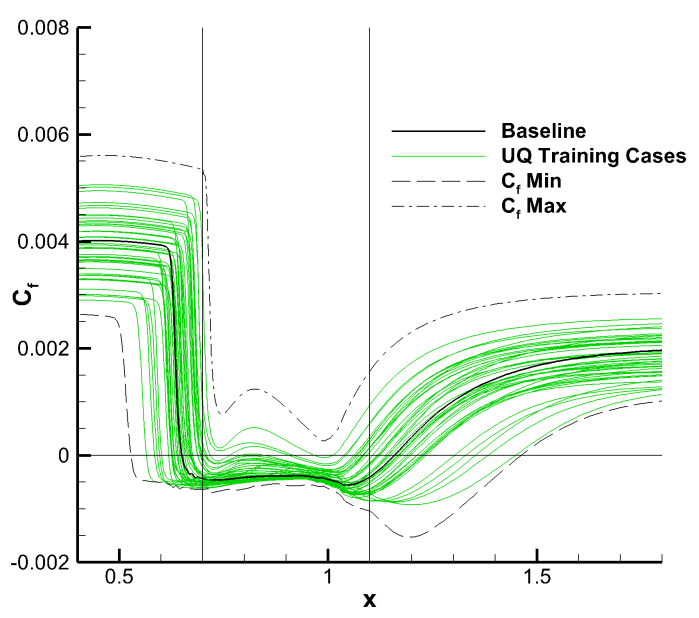

(e) $\boldsymbol{C}_{\boldsymbol{f}}$ versus $\boldsymbol{x}(\mathrm{SST})$.

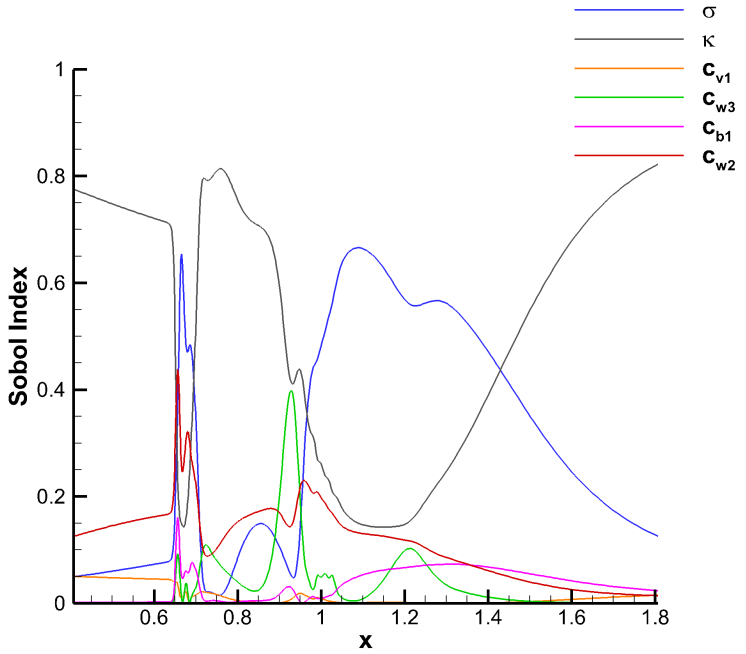

(b) Sobol indices for $\boldsymbol{C}_{\boldsymbol{f}}$ versus $\boldsymbol{x}(\mathrm{SA})$.

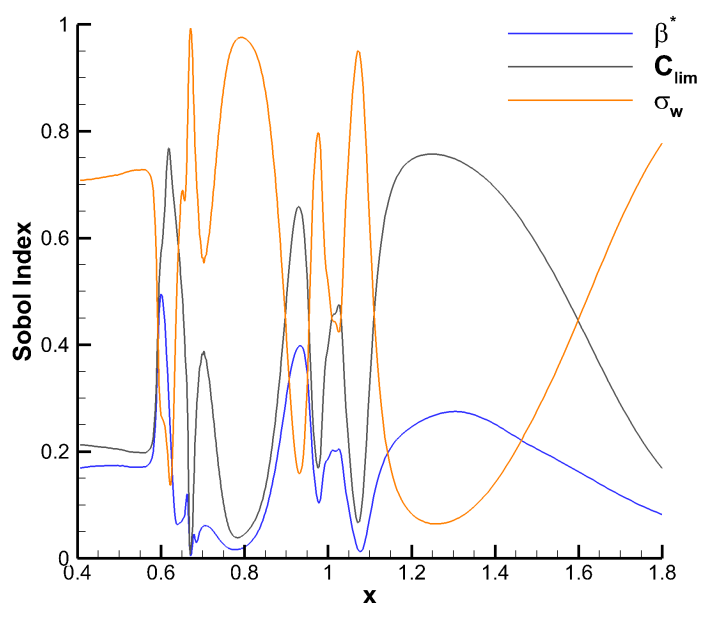

(d) Sobol indices for $\boldsymbol{C}_{\boldsymbol{f}}$ versus $\boldsymbol{x}$ (W2006).

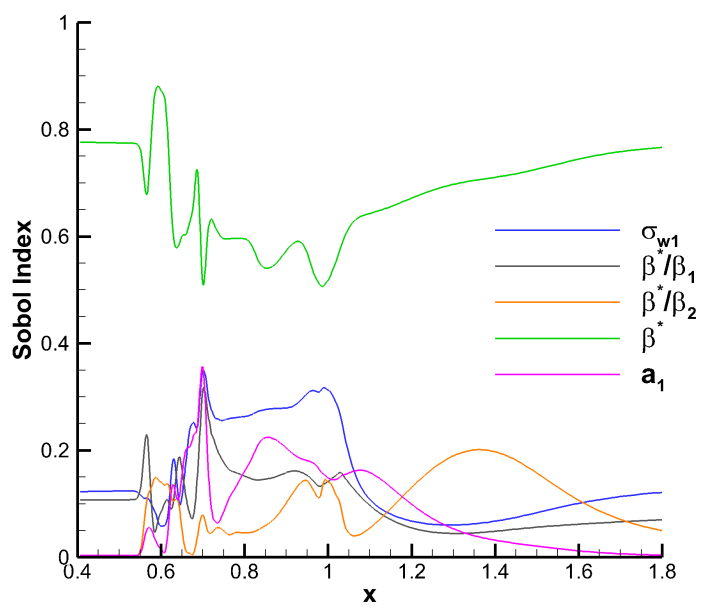

(f) Sobol indices for $\boldsymbol{C}_{\boldsymbol{f}}$ versus $\boldsymbol{x}$ (SST).

Figure 7. Skin friction coefficient results for transonic bump. 
Table 13. Sobol Indices of Closure Coefficients for $C_{L}$ of RAE 2822 .

\begin{tabular}{cccccc}
\hline \multicolumn{2}{c}{ SA } & \multicolumn{2}{c}{ W2006 } & \multicolumn{2}{c}{ SST } \\
Coefficient & Sobol Index & Coefficient & Sobol Index & Coefficient & Sobol Index \\
\hline$\kappa$ & $5.07 \times 10^{-1}$ & $C_{\text {lim }}$ & $8.55 \times 10^{-1}$ & $\beta^{*} / \beta_{2}$ & $4.22 \times 10^{-1}$ \\
$c_{t 4}$ & $2.34 \times 10^{-1}$ & $\beta^{*}$ & $9.57 \times 10^{-2}$ & $a_{1}$ & $4.13 \times 10^{-1}$ \\
$c_{t 3}$ & $1.82 \times 10^{-1}$ & $\sigma_{w}$ & $4.23 \times 10^{-2}$ & $\beta^{*}$ & $2.50 \times 10^{-1}$ \\
$c_{v 1}$ & $7.30 \times 10^{-2}$ & $\kappa$ & $1.83 \times 10^{-2}$ & $\sigma_{w 2}$ & $2.37 \times 10^{-2}$ \\
$c_{b 1}$ & $3.18 \times 10^{-2}$ & $\beta^{*} / \beta_{0}$ & $1.27 \times 10^{-2}$ & $\sigma_{w 1}$ & $1.64 \times 10^{-2}$ \\
$c_{w 2}$ & $1.78 \times 10^{-2}$ & $\sigma_{k}$ & $1.19 \times 10^{-2}$ & $\beta^{*} / \beta_{1}$ & $1.59 \times 10^{-2}$ \\
$\sigma$ & $1.55 \times 10^{-2}$ & & & $\sigma_{k 2}$ & $1.11 \times 10^{-2}$ \\
$c_{b 2}$ & $1.51 \times 10^{-2}$ & & & $\sigma_{k 1}$ & $9.95 \times 10^{-3}$ \\
$c_{w 3}$ & $9.52 \times 10^{-3}$ & & & $\kappa$ & $8.63 \times 10^{-3}$ \\
$c_{t 1}$ & $8.12 \times 10^{-3}$ & & & & \\
$c_{t 2}$ & $6.13 \times 10^{-3}$ & & & & \\
\hline
\end{tabular}

Table 14. Sobol Indices of Closure Coefficients for $C_{D_{\text {press }}}$.

\begin{tabular}{cccccc}
\hline \multicolumn{2}{c}{ SA } & \multicolumn{2}{c}{ W2006 } & \multicolumn{2}{c}{ SST } \\
Coefficient & Sobol Index & Coefficient & Sobol Index & Coefficient & Sobol Index \\
\hline$\sigma$ & $4.64 \times 10^{-1}$ & $C_{\text {lim }}$ & $6.62 \times 10^{-1}$ & $\beta^{*}$ & $6.30 \times 10^{-1}$ \\
$c_{w 2}$ & $2.13 \times 10^{-1}$ & $\sigma_{w}$ & $2.14 \times 10^{-1}$ & $\beta^{*} / \beta_{2}$ & $2.36 \times 10^{-1}$ \\
$c_{b 1}$ & $1.91 \times 10^{-1}$ & $\beta^{*}$ & $1.71 \times 10^{-1}$ & $a_{1}$ & $1.34 \times 10^{-1}$ \\
$c_{t 4}$ & $7.10 \times 10^{-2}$ & $\beta^{*} / \beta_{0}$ & $4.81 \times 10^{-3}$ & $\beta^{*} / \beta_{1}$ & $3.22 \times 10^{-2}$ \\
$c_{v 1}$ & $2.90 \times 10^{-2}$ & $\kappa$ & $2.46 \times 10^{-3}$ & $\sigma_{w 1}$ & $1.18 \times 10^{-2}$ \\
$\kappa$ & $2.74 \times 10^{-2}$ & $\sigma_{k}$ & $2.32 \times 10^{-3}$ & $\sigma_{w 2}$ & $1.11 \times 10^{-2}$ \\
$c_{t 3}$ & $2.07 \times 10^{-2}$ & & & $\sigma_{k 2}$ & $4.99 \times 10^{-3}$ \\
$c_{b 2}$ & $1.25 \times 10^{-2}$ & & & $\sigma_{k 1}$ & $4.71 \times 10^{-3}$ \\
$c_{t 2}$ & $7.16 \times 10^{-3}$ & & & $\kappa$ & $1.95 \times 10^{-3}$ \\
$c_{t 1}$ & $5.01 \times 10^{-3}$ & & & & \\
$c_{w 3}$ & $3.90 \times 10^{-3}$ & & & & \\
\hline
\end{tabular}

$\beta^{*}$, and $\sigma_{w}$. The order of importance is different for $C_{L}, C_{D_{\mathrm{press}}}$, and $C_{D_{\mathrm{sf}}}$, but the important coefficients are the same for each quantity.

For the SST model, the largest contributors to uncertainty in $C_{L}$ are $\beta^{*} / \beta_{2}, a_{1}$, and $\beta^{*}$. The largest contributors to uncertainty in $C_{D_{\text {press }}}$ are $\beta^{*}, \beta^{*} / \beta_{2}, a_{1}$, and $\beta^{*} \beta_{1}$; and the largest contributors to uncertainty in $C_{D_{\mathrm{sf}}}$ are $\beta^{*}, \sigma_{w 1}$, and $\beta^{*} / \beta_{1}$.

Response surfaces for $C_{p}$ were generated at each $x$-location on the top and bottom surface of the airfoil so that the closure coefficients with significant contributions to $C_{p}$ at any given point in the flow could be identified. These plots are included in Fig. 8. In Fig. 8a, the largest contributor to uncertainty in $C_{p}$ for the majority of the $x$ domain in the SA model is $\kappa$. There are also spikes in the Sobol indices of $c_{t 3}, c_{t 4}$, and $\sigma$, indicating that these coefficients are also important in some parts of the flow; particularly, spikes appear near the shock on the upper surface and near the high-pressure region at the leading edge on the lower surface. In Fig. 8b, the uncertainty in $C_{p}$ for the W2006 model is almost completely dominated by $C_{\text {lim }}$, with only a few minor contributions from $\beta^{*}$ and $\sigma_{2}$. In Fig. 8c, the uncertainty in $C_{p}$ for the SST model is mostly attributed to $\beta^{*} / \beta_{2}, \beta^{*}$, and $a_{1}$. At $x \approx 0.6, \sigma_{w 1}$ also has a minor contribution.

In sum, seven closure coefficients were found to contribute significantly to at least one output quantity of interest for the SA model; three coefficients for the W2006 model; and five coefficients for the SST model. This information is summarized in Table 16. 


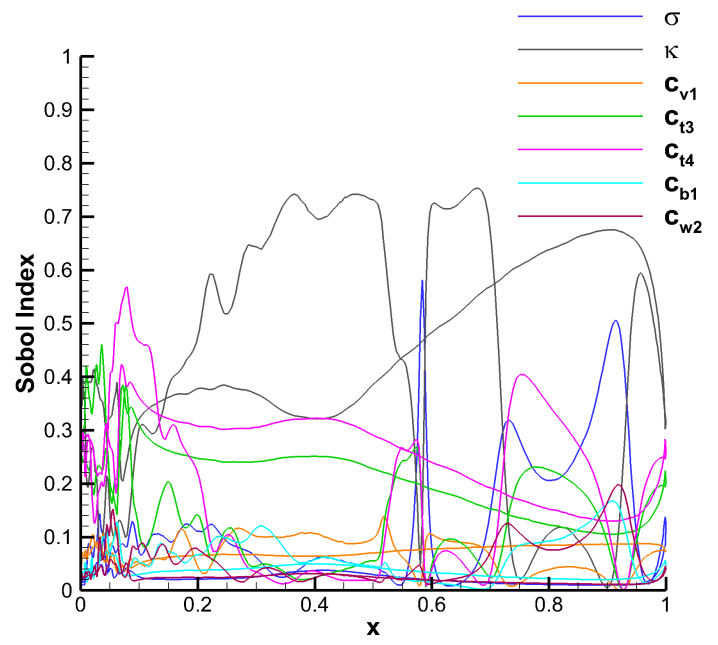

(a) Sobol indices for $\boldsymbol{C}_{\boldsymbol{p}}$ versus $\boldsymbol{x}$ (SA).

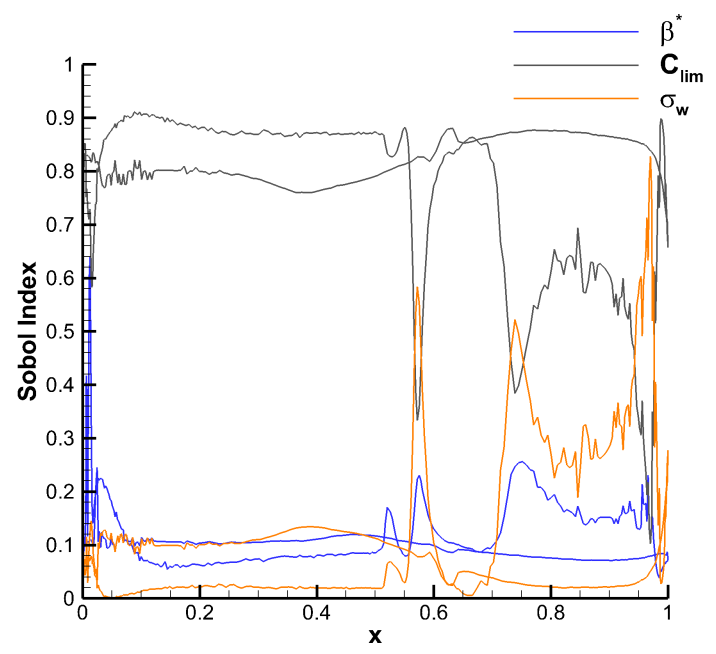

(b) Sobol indices for $\boldsymbol{C}_{\boldsymbol{p}}$ versus $\boldsymbol{x}$ (W2006).

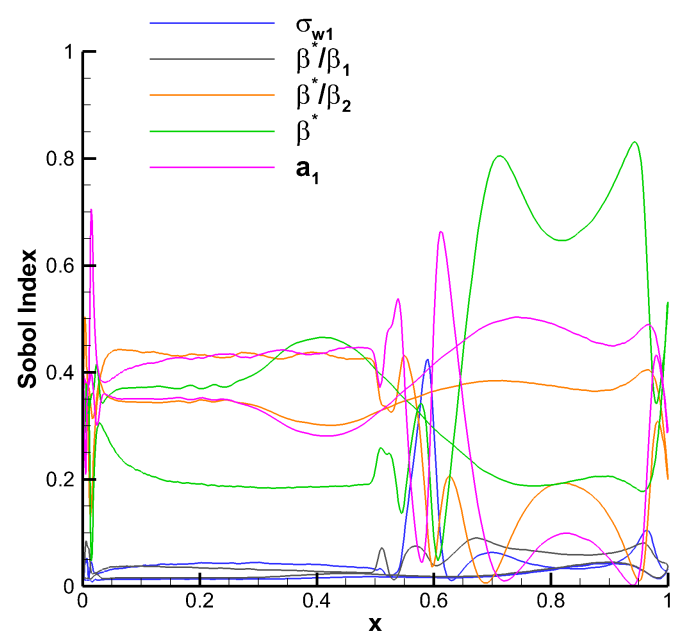

(c) Sobol indices for $\boldsymbol{C}_{\boldsymbol{p}}$ versus $\boldsymbol{x}$ (SST).

Figure 8. Sobol index distributions for RAE 2822 airfoil (Original Analyses).

19 of 26 
Table 15. Sobol Indices of Closure Coefficients for $C_{D_{\mathrm{sf}}}$.

\begin{tabular}{cccccc}
\hline \multicolumn{2}{c}{ SA } & \multicolumn{2}{c}{ W2006 } & \multicolumn{2}{c}{ SST } \\
Coefficient & Sobol Index & Coefficient & Sobol Index & Coefficient & Sobol Index \\
\hline$\kappa$ & $5.65 \times 10^{-1}$ & $\sigma_{w}$ & $6.41 \times 10^{-1}$ & $\beta^{*}$ & $7.75 \times 10^{-1}$ \\
$\sigma$ & $2.36 \times 10^{-1}$ & $C_{\text {lim }}$ & $2.39 \times 10^{-1}$ & $\sigma_{w 1}$ & $1.22 \times 10^{-1}$ \\
$c_{w 2}$ & $8.50 \times 10^{-2}$ & $\beta^{*}$ & $1.56 \times 10^{-1}$ & $\beta^{*} / \beta_{1}$ & $9.50 \times 10^{-2}$ \\
$c_{t 4}$ & $4.30 \times 10^{-2}$ & $\kappa$ & $1.07 \times 10^{-2}$ & $a_{1}$ & $9.84 \times 10^{-3}$ \\
$c_{t 3}$ & $3.58 \times 10^{-2}$ & $\sigma_{k}$ & $9.71 \times 10^{-3}$ & $\beta^{*} / \beta_{2}$ & $5.32 \times 10^{-3}$ \\
$c_{v 1}$ & $3.06 \times 10^{-2}$ & $\beta^{*} / \beta_{0}$ & $3.59 \times 10^{-3}$ & $\sigma_{w 2}$ & $1.17 \times 10^{-3}$ \\
$c_{b 1}$ & $1.97 \times 10^{-2}$ & & & $\sigma_{k 1}$ & $8.58 \times 10^{-4}$ \\
$c_{b 2}$ & $1.65 \times 10^{-3}$ & & & $\sigma_{k 2}$ & $8.34 \times 10^{-4}$ \\
$c_{t 1}$ & $1.36 \times 10^{-3}$ & & & $\kappa$ & $1.30 \times 10^{-4}$ \\
$c_{w 3}$ & $1.17 \times 10^{-3}$ & & & & \\
$c_{t 2}$ & $6.17 \times 10^{-4}$ & & & & \\
\hline
\end{tabular}

Table 16. Closure Coefficients with Significant Contributions to RAE 2822.

\begin{tabular}{ccc}
\hline SA & W2006 & SST \\
\hline$\sigma$ & $\beta^{*}$ & $\sigma_{w 1}$ \\
$\kappa$ & $C_{l i m}$ & $\beta^{*} / \beta_{1}$ \\
$c_{v 1}$ & $\sigma_{w}$ & $\beta^{*} / \beta_{2}$ \\
$c_{t 3}$ & & $\beta^{*}$ \\
$c_{t 4}$ & & $a_{1}$ \\
$c_{b 1}$ & & \\
$c_{w 2}$ & & \\
\hline
\end{tabular}

\section{V.D. RAE 2822 Airfoil - Reduced Dimensionality Analyses}

As with the transonic bump, a reduced-dimensionality analysis was performed on the RAE 2822 for each of the turbulence models to further improve the accuracy of the response surfaces used in the uncertainty analyses. A baseline case was run with each of the original, unchanged turbulence models for the RAE 2822 airfoil. The response surfaces of drag coefficient and lift coefficient for the RAE 2822 were verified in the same manner as the response surfaces of the drag coefficient of the transonic bump; the minimum and maximum values of $C_{D}$ and $C_{L}$ were first calculated from the response surfaces, and then CFD simulations were run by using the closure coefficients that produced these minimum and maximum values. The comparison between the response surfaces and CFD is summarized in Tables 17 and 18. All of the $C_{D}$ response surfaces are within $4.37 \%$ or less of the CFD, and the $C_{L}$ response surfaces are within $1.18 \%$ or less of the CFD.

The Sobol indices for $C_{L}, C_{D_{\text {press }}}$, and $C_{D_{\mathrm{sf}}}$ agree well with the Sobol indices in the original analyses. The results for the reduced-dimensionality analyses are included in Tables 19, 20, and 21. As with the transonic bump, the ranking of importance of the closure coefficients for each quantity is mostly the same between

Table 17. RAE 2822 Drag Coefficient Results, in Drag Counts (RDA).

\begin{tabular}{cccccccc}
\hline & Baseline $C_{D}$ & \multicolumn{3}{c}{ Min $C_{D}$} & \multicolumn{3}{c}{ Max $C_{D}$} \\
Model & CFD & Resp. Surf. & CFD & Difference & Resp. Surf. & CFD & Difference \\
\hline SA & 146.0 & 134.7 & 135.8 & $0.81 \%$ & 152.8 & 151.2 & $1.06 \%$ \\
W2006 & 142.3 & 130.2 & 128.5 & $1.32 \%$ & 144.4 & 144.4 & $0.00 \%$ \\
SST & 128.5 & 107.2 & 112.1 & $4.37 \%$ & 183.0 & 182.8 & $0.11 \%$ \\
\hline
\end{tabular}


Table 18. RAE 2822 Lift Coefficient Results (RDA).

\begin{tabular}{cccccccc}
\hline & Baseline $C_{L}$ & \multicolumn{3}{c}{ Min $C_{L}$} & \multicolumn{3}{c}{ Max $C_{L}$} \\
Model & CFD & Resp. Surf. & CFD & Difference & Resp. Surf. & CFD & Difference \\
\hline SA & 0.744 & 0.735 & 0.739 & $0.57 \%$ & 0.769 & 0.764 & $0.69 \%$ \\
W2006 & 0.744 & 0.733 & 0.738 & $0.68 \%$ & 0.749 & 0.748 & $0.13 \%$ \\
SST & 0.744 & 0.710 & 0.719 & $1.18 \%$ & 0.778 & 0.774 & $0.45 \%$ \\
\hline
\end{tabular}

Table 19. Sobol Indices of Closure Coefficients for $C_{L}$ of RAE 2822 (RDA).

\begin{tabular}{cccccc}
\hline \multicolumn{2}{c}{ SA } & \multicolumn{2}{c}{ W2006 } & \multicolumn{2}{c}{ SST } \\
Coefficient & Sobol Index & Coefficient & Sobol Index & Coefficient & Sobol Index \\
\hline$\kappa$ & $5.09 \times 10^{-1}$ & $C_{\text {lim }}$ & $8.96 \times 10^{-1}$ & $a_{1}$ & $5.21 \times 10^{-1}$ \\
$c_{t 4}$ & $2.18 \times 10^{-1}$ & $\beta^{*}$ & $9.93 \times 10^{-2}$ & $\beta^{*} / \beta_{2}$ & $3.27 \times 10^{-1}$ \\
$c_{t 3}$ & $1.81 \times 10^{-1}$ & $\sigma_{w}$ & $1.84 \times 10^{-2}$ & $\beta^{*}$ & $2.81 \times 10^{-1}$ \\
$c_{v 1}$ & $1.03 \times 10^{-1}$ & & & $\beta^{*} / \beta_{1}$ & $1.10 \times 10^{-1}$ \\
$\sigma$ & $2.83 \times 10^{-2}$ & & & $\sigma_{w 1}$ & $4.48 \times 10^{-2}$ \\
$c_{b 1}$ & $2.60 \times 10^{-2}$ & & & & \\
$c_{w 2}$ & $2.23 \times 10^{-2}$ & & & & \\
\hline
\end{tabular}

the original and reduced-dimensionality analyses. Even when the ranking of importance is not the same, the actual numeric values of the Sobol indices in the reduced-dimensionality analyses are still reasonably close to their corresponding values in the original analyses.

Pressure coefficient plots were generated for each turbulence model. Each of the plots contains data for the baseline case; UQ training cases (green lines); the minimum and maximum $C_{p}$ bounds; and experimental data from Cook et al. ${ }^{9}$ Cook et al. did not report any experimental uncertainty, so the experimental results are included as reference only. The $C_{p}$ versus $x$ plots are included in Figs. 9a, 9c, and 9e; and the Sobol indices for $C_{p}$ versus $x$ are included in Figs. 9b, 9d, and 9f. For clarity, only the Sobol indices of coefficients with significant contributions to uncertainty in $C_{p}$ are shown in the figures. Similarly to the transonic bump, the minimum and maximum $C_{p}$ curves Figs. 9a, 9c, and 9e are piece-wise combinations of the NIPC response surfaces and cases run at the corners of the hypercubes defined by the epistemic bounds of the closure coefficients in the shock region.

Based on the reduced-dimensionality results, all of the coefficients found to be significant to the RAE 2822 airfoil in Section V.C are indeed significant to their respective turbulence models. Of the three models, the SST model is most sensitive to changes in its closure coefficients, as evidenced by its larger variations in $C_{L}, C_{D_{\text {press }}}$, and $C_{D_{\mathrm{sf}}}$, and by its larger solution band for $C_{p}$ compared to the SA and W2006 models. The SA and W2006 models are roughly equally sensitive to changes in their closure coefficients, with the latter particularly insensitive. All of the models are most sensitive to changes in their closure coefficients in the shock region; everywhere else, there is very little uncertainty in $C_{p}$.

\section{V.E. Comparison of Uncertainty Quantification Results Between the Two CFD Cases}

The uncertainty quantification and sensitivity results of the axisymmetric transonic bump and RAE 2822 airfoil are consistent with one another. For the W2006 and SST models, every closure coefficient which is significant to at least one output quantity of interest for the transonic bump is also significant to at least one output quantity of interest for the RAE 2822 and vice versa. This agreement is nearly observed by the SA model as well, with only one coefficient significant to the transonic bump but not the RAE 2822, and only two coefficients significant to the RAE 2822, but not the transonic bump. A summary of all of the significant closure coefficients is included in Fig. 10.

Compared to the transonic bump results, the RAE 2822 results are much less sensitive to changes in all three turbulence models' closure coefficients. The same trends still apply however; the SST model is most sensitive to changes in its closure coefficients, and the SA and W2006 models are roughly equally sensitive 


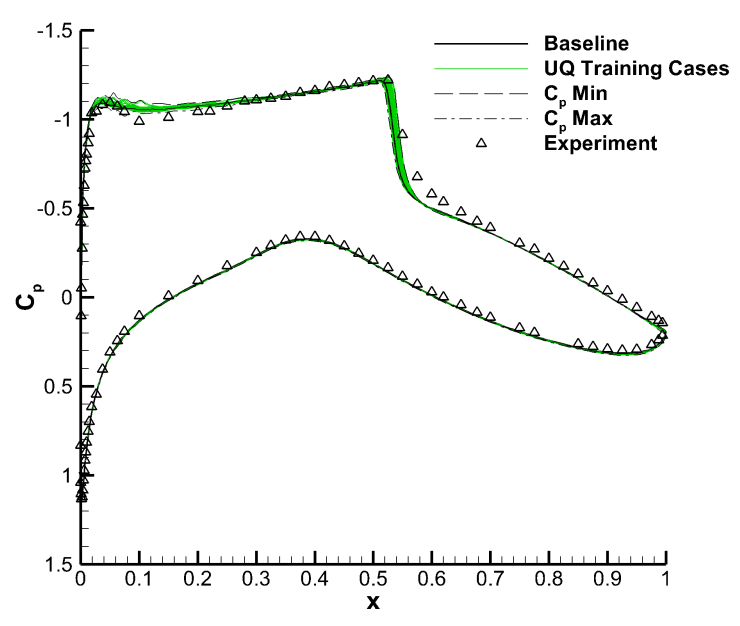

(a) $\boldsymbol{C}_{\boldsymbol{p}}$ versus $\boldsymbol{x}(\mathrm{SA})$.

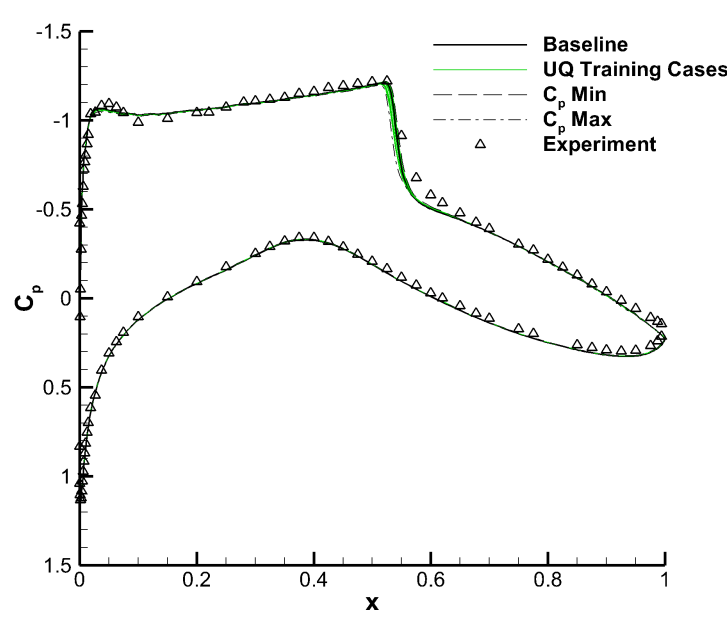

(c) $\boldsymbol{C}_{\boldsymbol{p}}$ versus $\boldsymbol{x}$ (W2006).

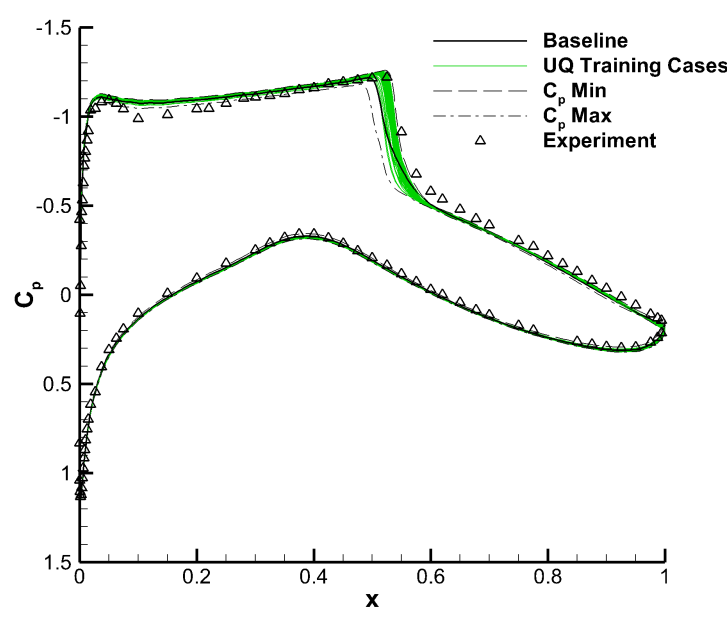

(e) $\boldsymbol{C}_{\boldsymbol{p}}$ versus $\boldsymbol{x}$ (SST).

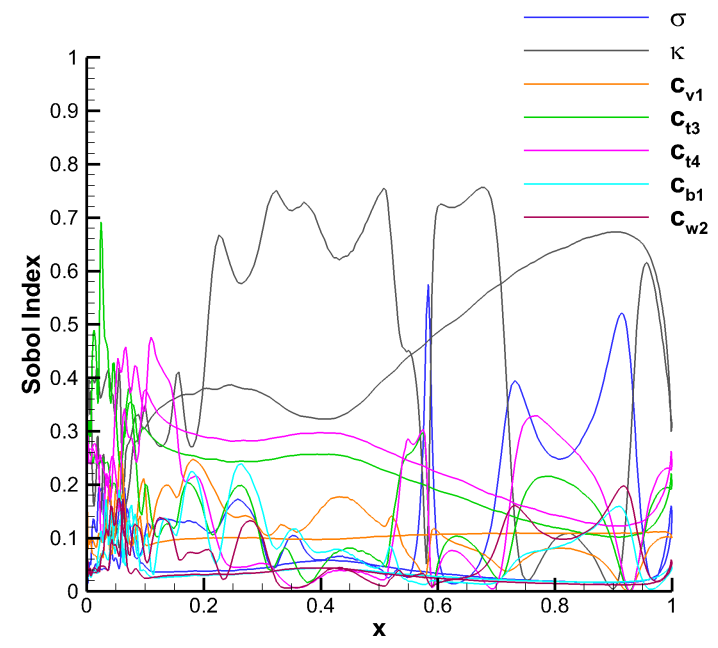

(b) Sobol indices for $\boldsymbol{C}_{\boldsymbol{p}}$ versus $\boldsymbol{x}$ (SA).

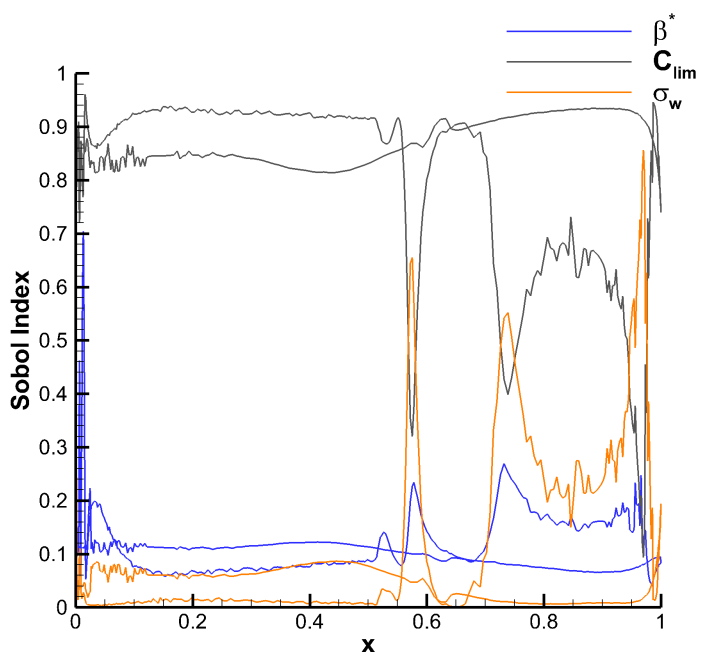

(d) Sobol indices for $\boldsymbol{C}_{\boldsymbol{p}}$ versus $\boldsymbol{x}$ (W2006).

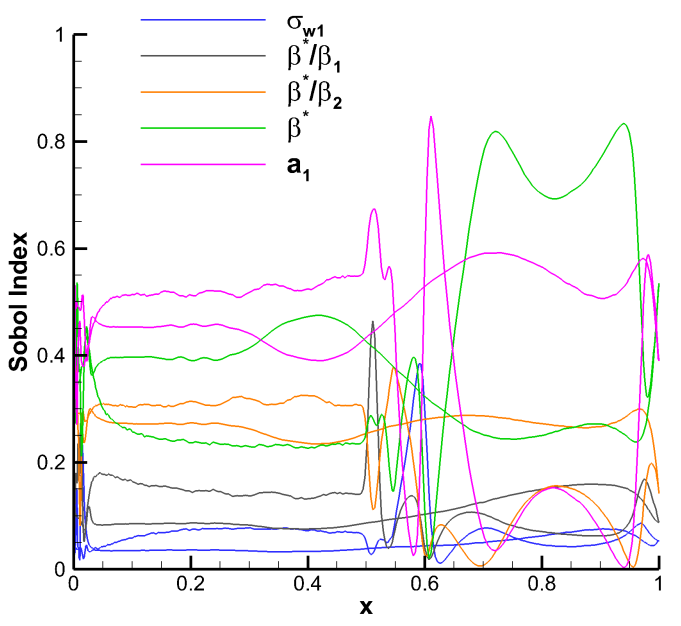

(f) Sobol indices for $\boldsymbol{C}_{\boldsymbol{p}}$ versus $\boldsymbol{x}$ (SST).

Figure 9. Pressure coefficient results for RAE 2822.

\section{2 of 26}


Table 20. Sobol Indices of Closure Coefficients for $C_{D_{\text {press }}}$ of RAE 2822 (RDA).

\begin{tabular}{cccccc}
\hline \multicolumn{2}{c}{ SA } & \multicolumn{2}{c}{ W2006 } & \multicolumn{2}{c}{ SST } \\
Coefficient & Sobol Index & Coefficient & Sobol Index & Coefficient & Sobol Index \\
\hline$\sigma$ & $4.55 \times 10^{-1}$ & $C_{\text {lim }}$ & $7.01 \times 10^{-1}$ & $\beta^{*}$ & $6.64 \times 10^{-1}$ \\
$c_{b 1}$ & $2.24 \times 10^{-1}$ & $\beta^{*}$ & $1.82 \times 10^{-1}$ & $\beta^{*} / \beta_{2}$ & $2.02 \times 10^{-1}$ \\
$c_{w 2}$ & $2.23 \times 10^{-1}$ & $\sigma_{w}$ & $1.64 \times 10^{-1}$ & $a_{1}$ & $1.77 \times 10^{-1}$ \\
$c_{t 4}$ & $5.26 \times 10^{-2}$ & & & $\beta^{*} / \beta_{1}$ & $5.15 \times 10^{-2}$ \\
$\kappa$ & $4.27 \times 10^{-2}$ & & & $\sigma_{w 1}$ & $2.19 \times 10^{-2}$ \\
$c_{v 1}$ & $3.19 \times 10^{-2}$ & & & & \\
$c_{t 3}$ & $2.17 \times 10^{-2}$ & & & & \\
\hline
\end{tabular}

Table 21. Sobol Indices of Closure Coefficients for $C_{D_{\mathrm{sf}}}$ of RAE 2822 (RDA).

\begin{tabular}{cccccc}
\hline \multicolumn{2}{c}{ SA } & \multicolumn{2}{c}{ W2006 } & \multicolumn{2}{c}{ SST } \\
Coefficient & Sobol Index & Coefficient & Sobol Index & Coefficient & Sobol Index \\
\hline$\kappa$ & $5.69 \times 10^{-1}$ & $\sigma_{w}$ & $6.92 \times 10^{-1}$ & $\beta^{*}$ & $7.91 \times 10^{-1}$ \\
$\sigma$ & $2.33 \times 10^{-1}$ & $C_{l i m}$ & $2.10 \times 10^{-1}$ & $\sigma_{w 1}$ & $1.15 \times 10^{-1}$ \\
$c_{w 2}$ & $8.30 \times 10^{-2}$ & $\beta^{*}$ & $1.45 \times 10^{-1}$ & $\beta^{*} / \beta_{1}$ & $9.10 \times 10^{-2}$ \\
$c_{t 4}$ & $4.25 \times 10^{-2}$ & & & $a_{1}$ & $1.09 \times 10^{-2}$ \\
$c_{v 1}$ & $4.05 \times 10^{-2}$ & & & $\beta^{*} / \beta_{2}$ & $3.68 \times 10^{-3}$ \\
$c_{t 3}$ & $3.52 \times 10^{-2}$ & & & & \\
$c_{b 1}$ & $1.89 \times 10^{-2}$ & & & & \\
\hline
\end{tabular}

to changes in their closure coefficients. Uncertainties in $C_{p}$ are particularly large in the shock region of each flow for all three turbulence models.

The information presented in this paper is expected to be of interest to the CFD community because it demonstrates that large changes in turbulence model behavior can be observed by changing specific sets of closure coefficients. In particular, there are two main results that can enable the advancement of turbulence models used in RANS simulations. The first result is that if improved matching between CFD and experiments (i.e., the data representing the real physical phenomena) is desired, the Sobol indices presented in this work for certain output quantities of interest give clues for which closure coefficients to modify first. The second result is that if the aerospace community can reduce the uncertainty in only a certain set of closure coefficients (whether through experimentation, LES/DES, etc.), the uncertainty in turbulence model predictions can be greatly reduced. For example, the uncertainty in the value of Von Kármán's constant in the SA model was responsible for approximately $85 \%$ of the uncertainty in $C_{D}$ of the axisymmetric transonic bump. If more information were known about the true value of $\kappa$, the results of the SA model would be much less uncertain.

If improved accuracy is desired, a good starting point may be to first consider the integrated quantities; that is, $C_{D}$ for the axisymmteric transonic bump, and $C_{L}, C_{D_{\text {press }}}$, and $C_{D_{\text {sf }}}$ for the RAE 2822 airfoil. Referring back to Tables 11, 19, 20, and 21, a small number of closure coefficients can be identified for each turbulence model which consistently contribute to very large amounts to uncertainty in the output quantities of interest. These coefficients include $\kappa$ and $\sigma$ for the SA model; $C_{l i m}$ and $\sigma_{w}$ for the W2006 model; and $\beta^{*}$ for the SST model. The authors suggest that these coefficients be considered first in future refinements of the turbulence models discussed here.

\section{Conclusions and Future Work}

Two detailed uncertainty quantification studies focusing on turbulence model closure coefficients were performed on an axisymmetric transonic bump at zero degrees angle of attack and the RAE 2822 transonic airfoil at a lift coefficient of 0.744 . The transonic bump has freestream $M=0.875$ and $R e=2.763 \times 10^{6}$, 


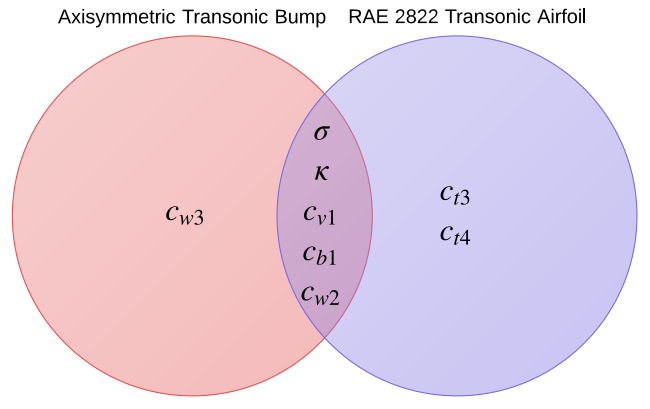

(a) SA - Significant closure coefficients.

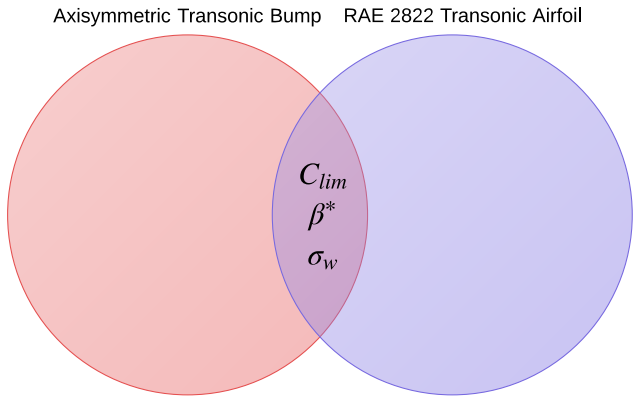

(b) W2006 - Significant closure coefficients.

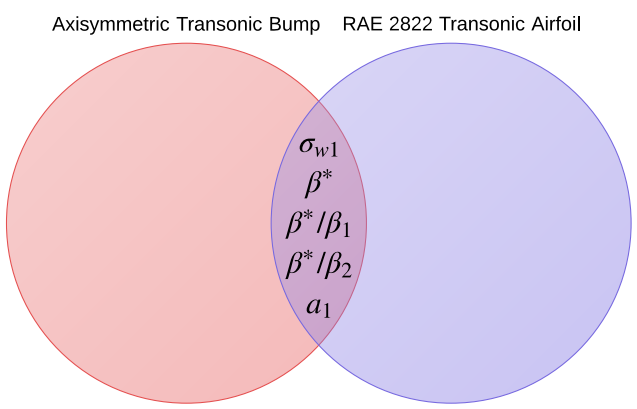

(c) SST - Significant closure coefficients.

Figure 10. Closure coefficients significant to uncertainty in at least one output quantity of interest for each CFD problem and for each turbulence model.

and the RAE 2822 has freestream $M=0.729$ and $R e=6.5 \times 10^{6}$. The turbulence models considered were the Spalart-Allmaras Model, Wilcox (2006) k- $\omega$ Model, and Menter Shear-Stress Transport Model. The uncertainty quantification employed stochastic expansions based on non-intrusive polynomial chaos as an efficient means of uncertainty propagation. The drag coefficient, wall pressure and skin friction coefficient distributions, and shock-induced separation bubble size were considered as uncertain outputs for the transonic bump. The lift coefficient, pressure and skin friction components of drag coefficient, and wall pressure coefficient distribution were considered as uncertain outputs for the RAE 2822. Sobol indices were used to rank the relative contributions of each closure coefficient to the total uncertainty in the output quantities of interest. This information can be used a potential starting point in the future advancement of the turbulence models considered here, and it is a first step towards quantifying model-form uncertainty.

Based on the results, the following conclusions can be made: For the SA model, the coefficients that contribute most to uncertainty in the output quantities of interest are $\sigma, \kappa, c_{v 1}, c_{b 1}, c_{w 2}, c_{t 3}, c_{t 4}$, and $c_{w 3}$. For the W2006 model, the coefficients that contribute most to uncertainty in the output quantities of interest are $\beta^{*}, C_{\text {lim }}$, and $\sigma_{w}$. For the SST model, the coefficients that contribute most to uncertainty in the output quantities of interest are $\sigma_{w 1}, \beta^{*} / \beta_{1}, \beta^{*} / \beta_{2}, \beta^{*}$, and $a_{1}$. With the exception of $c_{w 3}, c_{t 3}$, and $c_{t 4}$ in the SA model, all other closure coefficients were found to be significant to both the axisymmetric transonic bump and RAE 2822 transonic airfoil.

In the future refinement of the models discussed here, the authors suggest that studies should focus on increasing knowledge of appropriate values for $\kappa$ and $\sigma$ for the SA model; $C_{\text {lim }}$ and $\sigma_{w}$ for the W2006 model; and $\beta^{*}$ for the SST model. Improved knowledge of the values of these particular closure coefficients 
will have the largest impact on reducing the uncertainty in integrated quantities of interest for the RANS simulations of wall-bounded transonic flows. Future work should also focus on determining why certain closure coefficients contribute more or less to uncertainty at different flow locations. For example, research should be done to determine why $\sigma$ in the SA model becomes important to $C_{f}$ for the transonic bump after the reattachment point, but not before. Finally, future work should include more CFD problems, particularly in three dimensions.

\section{Acknowledgments}

The authors would like to give special thanks to Philippe Spalart, David Wilcox, Florian Menter, and Gary Coleman for their contributions to this research. The support for the first and the third authors for this research is provided under NASA Grant NNX14AN17A (Mujeeb Malik, program monitor). The first author would also like to thank the NASA Langley Research Center and the 2014 LARSS program for additional support.

\section{References}

${ }^{1}$ Spalart, P. R. and Allmaras, S. R., "A One-Equation Turbulence Model for Aerodynamic Flows," Recherche Aerospatiale, No. 1, 1994, pp. 5-21.

${ }^{2}$ Wilcox, D. C., "Formulation of the k-omega Turbulence Model Revisited," AIAA Journal, Vol. 46, No. 11, 2008, pp. $2823-2828$.

${ }^{3}$ Menter, F. R., "Two-Equation Eddy-Viscosity Turbulence Models for Engineering Applications," AIAA Journal, Vol. 32, No. 8, August 1994, pp. 1598-1605.

${ }^{4}$ Bachalo, W. D. and Johnson, D. A., "Transonic, Turbulent Boundary-Layer Separation Generated on an Axisymmetric Flow Model," AIAA Journal, Vol. 24, No. 3, 1986, pp. 437-443.

${ }^{5}$ Hosder, S., Walters, R. W., and Balch, M., "Point-Collocation Nonintrusive Polynomial Chaos Method for Stochastic Computational Fluid Dynamics," AIAA Journal, Vol. 48, No. 12, December 2010.

${ }^{6}$ Sudret, B., "Global sensitivity analysis using polynomial chaos expansion," Reliability Engineering and System Safety, Vol. 93, No. 7, 2008, pp. 964-979.

${ }^{7}$ Biedron, R. T., Derlaga, J. M., Gnoffo, P. A., Hammond, D. P., Jones, W. T., Kleb, B., Lee-Rausch, E. M., Nielsen, E. J., Park, M. A., Rumsey, C. L., Thomas, J. L., and Wood, W. A., "FUN3D Manual: 12.4," NASA/TM-2014-218179, March 2014.

8 "NASA Langley Research Center Turbulence Modeling Resource," http://turbmodels.larc.nasa.gov [retrieved April 2015].

${ }^{9}$ Cook, P.H., McDonald, M.A., and Firmin, M.C.P., "Aerofoil RAE 2822 - Pressure Distributions, and Boundary Layer and Wake Measurements," Experimental Data Base for Computer Program Assessment, AGARD Report AR $138,1979$.

${ }^{10}$ Nee, V. W. and Kovasznay, L. S. G., "The Calculation of the Incompressible Turbulent Boundary Layer by a Simple Theory," Physics of Fluids, Vol. 12, 1968, pp. 473.

${ }^{11}$ Bailey, S. C. C., Vallikivi, M., Hultmark, M., and Smits, A. J., "Estimating the value of von Kármán's constant in turbulent pipe flow," Journal of Fluid Mechanics, Vol. 749, 2014, pp. 79-98.

${ }^{12}$ Email correspondence between Philippe Spalart and John Schaefer, June-December 2014.

${ }^{13}$ Wilcox, D. C., Turbulence Modeling for CFD, 3rd edition, DCW Industries, Inc., La Cañada CA, 2006.

${ }^{14}$ Email correspondence between David Wilcox and John Schaefer, June 2014.

${ }^{15}$ Georgiadis, N. J. and Yoder, D. A., "Recalibration of the Shear Stress Transport Model to Improve Calculation of Shock Separated Flows," AIAA Paper 2013-0685, January 2013.

${ }^{16}$ Email correspondence between Florian Menter and John Schaefer, June 2014.

${ }^{17}$ West, T. K. and Hosder, S., "Uncertainty Quantification of Hypersonic Reentry Flows with Sparse Sampling and Stochastic Expansions," Journal of Spacecraft and Rockets, Vol. 52, No. 1, January-February 2015, pp. 120-133.

${ }^{18}$ Ghanem, R. G. and Spanos, P. D., "Stochastic Finite Elements: A Spectral Approach," Springer-Verlag, New York, 1991.

${ }^{19}$ Eldred, M. S., "Recent Advances in Non-Intrusive Polynomial Chaos and Stochastic Collocation Methods for Uncertainty Analysis and Design," AIAA Paper 2009-2274, May 2009.

${ }^{20}$ Hosder, S., Walters, R. W., and Balch, M., "Efficient Sampling for Non-Intrusive Polynomial Chaos Applications with Multiple Input Uncertain Variables," AIAA-Paper 2007-1939, April 2007.

${ }^{21}$ Crestaux, T., Maitre, O. L., and Martinez, J.-M, "Polynomial chaos expansion for sensitivity analysis," Reliability Engineering and System Safety, 2009.

${ }^{22}$ Ghaffari, S., Magin, T., and Iaccarino, G., "Uncertainty Quantification of Radiative Heat Flux Modeling for Titan Atmospheric Entry," AIAA Paper 2010-239, January 2010. 


\section{Appendix A: Sobol Index Derivation}

In this section, the derivation of Sobol indices is presented. First, the total variance, $D$, can be written in terms of the PCE as shown in Eq. (37).

$$
D=\sum_{j=1}^{P} \alpha_{j}^{2}(t, \vec{x})\left\langle\Psi_{j}^{2}(\vec{\xi})\right\rangle
$$

Then, as shown by Sudret ${ }^{6}$ and Crestaux et al. ${ }^{21}$ the total variance can be decomposed as:

$$
D=\sum_{i=1}^{i=n} D_{i}+\sum_{1 \leq i<j \leq n}^{i=n-1} D_{i, j}+\sum_{1 \leq i<j<k \leq n}^{i=n-2} D_{i, j, k}+\cdots+D_{1,2, \ldots, n}
$$

where the partial variances $\left(D_{i_{1}, \ldots, i_{s}}\right)$ are given by:

$$
D_{i_{1}, \ldots, i_{s}}=\sum_{\beta \in\left\{i_{1}, \ldots, i_{s}\right\}} \alpha_{\beta}^{2}\left\langle\Psi_{\beta}^{2}(\vec{\xi})\right\rangle, \quad 1 \leq i_{1}<\ldots<i_{s} \leq n
$$

Then the Sobol indices $\left(S_{i_{1} \cdots i_{s}}\right)$ are defined as,

$$
S_{i_{1} \cdots i_{s}}=\frac{D_{i_{1}, \ldots, i_{s}}}{D}
$$

which satisfy the following equation:

$$
\sum_{i=1}^{i=n} S_{i}+\sum_{1 \leq i<j \leq n}^{i=n-1} S_{i, j}+\sum_{1 \leq i<j<k \leq n}^{i=n-2} S_{i, j, k}+\cdots+S_{1,2, \ldots, n}=1.0
$$

The Sobol indices provide a sensitivity measure due to individual contribution from each input uncertain variable $\left(S_{i}\right)$, as well as the mixed contributions $\left(\left\{S_{i, j}\right\},\left\{S_{i, j, k}\right\}, \cdots\right)$. As shown by Sudret ${ }^{6}$ and Ghaffari et al., ${ }^{22}$ the total (combined) effect $\left(S_{T_{i}}\right)$ of an input parameter $i$ is defined as the summation of the partial Sobol indices that include the particular parameter:

$$
S_{T_{i}}=\sum_{L_{i}} \frac{D_{i_{1}, \ldots, i_{s}}}{D} ; \quad L_{i}=\left\{\left(i_{1}, \ldots, i_{s}\right): \exists k, 1 \leq k \leq s, i_{k}=i\right\}
$$

For example, with $n=3$, the total contribution to the overall variance from the first uncertain variable $(i=1)$ can be written as:

$$
S_{T_{1}}=S_{1}+S_{1,2}+S_{1,3}+S_{1,2,3}
$$

From these formulations, it can be seen that the Sobol indices can be used to provide a relative ranking of each input uncertainty to the overall variation in the output with the consideration of nonlinear correlation between input variables and output quantities of interest. 